\title{
Strong and fast rising pressure waves emitted by plasmonic vapor nanobubbles
}

\author{
Julien Lombard $\odot,{ }^{1}$ Julien Lam, ${ }^{2}$ François Detcheverry $\odot,{ }^{3}$ Thierry Biben, ${ }^{3}$ and Samy Merabia $\odot^{3, *}$ \\ ${ }^{1}$ Departamento de Física y Química Teórica, Facultad de Química, Universidad Nacional Autónoma de México, Mexico City 04510, Mexico \\ ${ }^{2}$ CEMES, CNRS, and Université de Toulouse, 29 rue Jeanne Marvig, 31055 Toulouse Cedex, France \\ ${ }^{3}$ Université Lyon, Université Claude Bernard Lyon 1, CNRS, Institut Lumière Matière, F-69622 Villeurbanne, France
}

(Received 10 December 2020; accepted 13 May 2021; published 22 June 2021)

\begin{abstract}
Plasmonic vapor nanobubbles are currently considered for a wide variety of applications ranging from solar energy harvesting and photoacoustic imaging to nanoparticle-assisted cancer therapy. Yet, due to their small size and unstable nature, their generation and consequences remain difficult to characterize. Here, building on a phase-field model, we report on the existence of strong pressure waves that are emitted when vapor nanobubbles first form around a laser-heated nanoparticle immersed in water and subsequently after bubble rebound. These effects are strongest when the fluid is locally brought high in its supercritical state, which may be realized with a short laser pulse. Because of the greatly out-of-equilibrium nature of nanobubble generation, the waves combine a high-pressure peak with a fast pressure rising time and propagate in water over micron distances, opening the way to induce spatially and temporally localized damage. Discussing the consequences on biological cell membranes, we conclude that acoustic-mediated perforation is more efficient than nanobubble expansion to breach the membrane. Our findings should serve as a guide for optimizing the thermoacoustic conversion efficiency of plasmonic vapor nanobubbles.
\end{abstract}

DOI: 10.1103/PhysRevResearch.3.023231

\section{INTRODUCTION}

Due to their wide range of applications in biomedicine, surface cleaning, and photoacoustic imaging [1,2], plasmonic vapor nanobubbles have received ever increasing attention from the scientific community. Their formation involves both the remarkable optical properties of metallic nanoparticles and the unique thermomechanical response of nanoscale vapor blankets. Nanoparticles offer not only versatility in optical absorption, controlled by the size, composition, and laser wavelength, but also biocompatibility and possible conjugation with antibodies for specific targeting [3].

When irradiated by strong laser pulses, metallic nanoparticles may be heated up by hundredths of a kelvin [4]. At the basis of this phenomenon are the interaction between the laser and the nanoparticle conduction electrons and its exaltation when the incident electromagnetic wavelength is close to the nanoparticle surface plasmon resonance, which lies in the visible range for common nanostructures [5]. Nanoparticles in solution thus behave as nanoscale hot spots for the liquid environment. In this unique situation, water may undergo a locally quick phase change, as its local temperature approaches the spinodal, around $550 \mathrm{~K}$ for water [6,7]. Indeed, at such small scales, the energy barriers for heterogeneous nucleation are so high that liquid water may be trapped in a metastable state up to temperatures much higher than the normal boiling

\footnotetext{
*samy.merabia@univ-lyon1.fr

Published by the American Physical Society under the terms of the Creative Commons Attribution 4.0 International license. Further distribution of this work must maintain attribution to the author(s) and the published article's title, journal citation, and DOI.
}

temperature, $375 \mathrm{~K}$ [8]. In this respect, vapor generation at the nanoscale shares similarities with the process of explosive boiling characterizing homogeneous nucleation [9-11]. Both phenomena are of an explosive nature as a result of the instability nature of the phase transition, in sharp contrast with the usual nucleation scenario [12,13]. Yet, these situations open new questions in liquid state physics at small scales [6,14-17], where phase changes are governed by nanoscale effects including Kapitza resistance and ballistic vapor transport [18]. In particular, the nanobubble threshold has been shown to be sensitive to, among others, the nanoparticle size [7,19,20], shape [21], surface coverage [22], laser pulse width [23], and presence of dissolved gas [15].

It has long been recognized that nanobubble generation around optically heated nano- and micro-objects [24] induces the emission of strong pressure waves [9-11]. One macroscopic consequence is a giant photomechanical effect [25], seen in interacting assemblies of nanoparticles with negative thermophoresis. At the microscopic scale, the acoustic emission concomitant to nanobubble generation opens the way to destruction of cancer cells at a subcellular level [26,27]. However, the very physical cause of cell membrane poration and eventual cell death remains debated. There are indeed two mechanisms according to which vapor nanobubbles may eventually cause cell destruction. In the first scenario, the acoustic pressure waves produced by water vaporization alter the cell membrane integrity [28]. In the second scenario, the expansion of plasmonic nanobubbles generates compressive strains of a few percent causing cell membrane breaching [27,29-34]. In deciding which scenario actually holds, a quantitative understanding of the phenomena induced by heated nanoparticles is essential. In contrast to nanobubble-based cell therapy, where mechanical damage is to be maximized, photoacoustic imaging seeks to minimize acoustic emission 
in its quest for a noninvasive and nondestructive technique [35]. This is only possible with a fundamental understanding of acoustic emission subsequent to explosive vapor formation around illuminated nano-objects.

Despite their importance for applications of photoacoustic conversion, experimental investigations of nanobubbleinduced pressure waves have remained scarce in the literature [36,37]. On the computational side, a body of work dealt with the modeling of photoacoustic effects under moderately intense laser excitation [38-41]. Relatively little attention has been paid to the case of photoacoustic effects induced by nanocavitation, with noticeable exceptions $[26,27,42-46]$ or in the context of laser-irradiated retinas [47]. However, most of these modeling studies rely on two simplifying assumptions. The first is the thermodynamic state of water following quick energy transfer from the particle: saturation is assumed for the liquid all the way to its vaporization. The second assumption concerns the nature of acoustic emission by nanobubbles, which is often modeled as a radiation effect. Whether these assumptions are warranted has remained untested so far.

In this study, we propose for nanobubble-induced acoustic emission a well-grounded approach that, in contrast with previous descriptions, does not rely on simplifying assumptions. We use a hydrodynamic phase-field model to predict bubble formation and pressure wave propagation around gold nanoparticles illuminated at a wavelength of $400 \mathrm{~nm}$ close to their surface plasmon resonance and characterize the pressure wave field generated by explosive vapor formation. Our simulations demonstrate that acoustic emission is a strongly nonequilibrium effect, resulting from the quick formation of a vapor/liquid interface under the very high temperature gradients generated at the nanoparticle/water interface. The result is not only a high peak in pressure, but also a short rising time to reach it, leading to a high growth rate of pressure. These effects are especially pronounced for short laser pulses, but long pulses may produce echoes of equal-amplitude acoustic waves. The assumptions that locally the thermodynamic state of water follows its saturation line and that acoustic emission is caused by bubble expansion cannot be retained in general, as they could actually severely underestimate the phenomena at play. As we illustrate by discussing acoustic-mediated cell membrane poration, the strong pressure waves irradiated by heated nano-objects have potentially powerful effects. Our findings should be helpful to optimize the practical applications of plasmonic vapor nanobubbles to cancer cell therapy.

The remainder of this article is organized as follows. We present in Sec. II our model for the nanoparticle and the fluid that surrounds it. Section III details our results on the generation and propagation of waves, the thermodynamics of the fluid, and the efficiency of the process. We discuss in Sec. IV an application to the mechanism of thermomechanical damage in membrane cells. A summary and some perspectives are given in Sec. V.

\section{MODEL}

The physical model has been presented elsewhere $[19,48]$. The reader interested in the results may skip this section and move directly to Sec. III. Generally speaking, the ap-

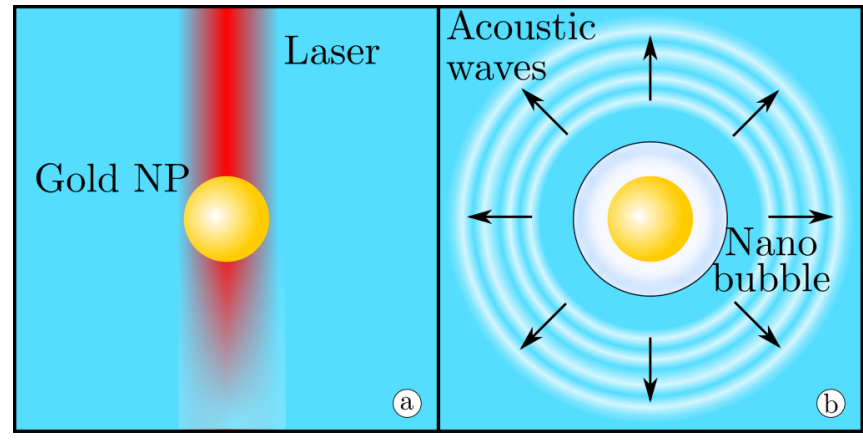

FIG. 1. Illustration of the situation considered: a gold nanoparticle in water is quickly heated by a laser pulse (a), generating a transient vapor nanobubble and the concomitant emission of pressure waves that propagate in the liquid (b).

proach is based on a diffuse interface model of the fluid accounting for phase transition and acoustic wave propagation. This type of model has been proposed and discussed in the literature [48-50] and applied successfully to shock formation following vapor nanobubble collapse [51,52]. Here, we consider acoustic emission following vapor nanobubble generation by heated nanoparticles. The model relies on the assumed symmetry of both the nanobubble generation and its dynamics. These two hypotheses are corroborated by the facts that (i) molecular dynamics and lattice Boltzmann simulations support the assumption that nanobubble generation is symmetrical $[20,53,54]$, and (ii) a stability analysis showed that for nanoparticle radii smaller than $100 \mathrm{~nm}$, the nanobubble retains its spherical shape during its growth [55].

\section{A. The nanoparticle}

The situation we aimed at modeling here, depicted in Fig. 1, is a gold nanoparticle immersed in water and illuminated by a laser pulse with a wavelength of $400 \mathrm{~nm}$, close to the surface plasmon resonance of the nanoparticle. As a result of the strong absorption, the temperature of gold electrons $T_{e}$ rises, which induces lattice vibrations through electronphonon coupling, with a relaxation time $\tau_{\mathrm{ep}} \simeq 7 \mathrm{ps}$. Since we consider laser pulses with a duration $\tau_{\mathrm{p}}$ of at least $10 \mathrm{ps}$, we can discard any electron-phonon disequilibrium inside the nanoparticle and assign to it a single temperature describing both the electronic and the vibrational degrees of freedom. Besides, the temperature within the nanoparticle is assumed to be uniform, a reasonable hypothesis owing to the large conductivity of the metal compared to that of liquid water.

Under these conditions, the temporal evolution of the nanoparticle temperature $T_{\mathrm{np}}$ is described by

$$
\begin{aligned}
V_{\mathrm{np}} C_{\mathrm{np}} \frac{d T_{\mathrm{np}}}{d t} & =F \sigma_{\mathrm{abs}} \frac{\Pi\left(t / \tau_{\mathrm{p}}\right)}{\tau_{\mathrm{p}}}-S_{\mathrm{np}} \phi, \\
\phi & =G\left(T_{\mathrm{np}}-T_{\mathrm{s}}\right) .
\end{aligned}
$$

$S_{\mathrm{np}}$ and $V_{\mathrm{np}}$ are the surface and volume of the nanoparticle, respectively, and $C_{\mathrm{np}}=2500 \mathrm{~kJ} / \mathrm{m}^{3} \mathrm{~K}$ is the gold specific heat. The interaction between laser and nanoparticle is described by the size-dependent absorption cross section $\sigma_{\text {abs }}(\lambda)$ as given by Mie theory [56]. The laser pulse has fluence $F$ and duration 
$\tau_{\mathrm{p}}$ and the gate function $\Pi(t)$ is unity for $0<t<1$ and 0 otherwise

The last term in Eq. (1) involves the flux $\phi$ of heat leaving the nanoparticle. In what follows, we consider that this flux is proportional to the difference between the nanoparticle temperature and the temperature $T_{\mathrm{s}}$ of the fluid at the surface [57-59], as prescribed by Eq. (2). The prefactor is the thermal boundary conductance $G$. It maintains a fixed value $G=90 \mathrm{MW} / \mathrm{m}^{2} / \mathrm{K}$, corresponding to a neutral interface (the equilibrium contact angle is around $90^{\circ}$ ). The practical implementation of this contact angle is fully described in Ref. [19].

\section{B. Governing equations for the fluid}

To model the fluid dynamics, we resort to a hydrodynamic phase-field model based on the free energy density, which solves continuum equations for the fluid dynamics based on a diffuse-interface model [60] and which has been successfully applied to address interfacial heat transport and boiling at the nanoscale $[49,61]$. The hydrodynamic conservation equations to describe the dynamics of the fluid around the nanoparticle are

$$
\begin{gathered}
\frac{\partial \rho}{\partial t}+\nabla \cdot(\rho \mathbf{v})=0, \\
m \rho(\mathbf{r})\left(\frac{\partial \mathbf{v}}{\partial t}+\mathbf{v} \cdot \nabla \mathbf{v}\right)=-\nabla \cdot(\mathbf{P}-\mathbf{D}), \\
m \rho(\mathbf{r}) c_{v}(\mathbf{r})\left(\frac{\partial T}{\partial t}+\mathbf{v} \cdot \nabla T\right) \\
=-l(\mathbf{r}) \nabla \cdot \mathbf{v}+\nabla \cdot(k(\mathbf{r}) \nabla T)+\mathbf{D}: \nabla v,
\end{gathered}
$$

where $\rho, \mathbf{v}$, and $T$ stand, respectively, for the number density, the velocity field, and the temperature field of the fluid; $m$ is the mass of a fluid molecule; $c_{v}$ and $k$ are the fluid specific heat and thermal conductivity; and $l=T(\partial P / \partial T)_{\rho}$ is the Clapeyron coefficient. Because these quantities depend on the local thermodynamic state, we have emphasized their spatial dependence on the position $\mathbf{r}$ in the fluid. Finally, $\mathbf{D}$ and $\mathbf{P}$ stand, respectively, for the dissipative stress tensor and pressure tensor. The symbol ":" indicates a double-dot product.

These equations are quite general and may be derived starting from the conservation of mass, linear momentum, and internal energy. The pressure tensor and the transport coefficients, however, depend on the specific model retained to describe the fluid. In the general heterogeneous and nonisothermal situation that we aim to describe, the pressure tensor appearing in Eq. (4) is not necessarily isotropic, and its general expression is given by the so-called Korteweg expression

$$
P_{\alpha \beta}=\left[P_{\text {bulk }}-w \rho \triangle \rho-\frac{w}{2}(\nabla \rho)^{2}\right] \delta_{\alpha \beta}+w \partial_{\alpha} \rho \partial_{\beta} \rho,
$$

where the bulk thermodynamic pressure $P_{\text {bulk }}$ is

$$
P_{\text {bulk }}=-\left(\frac{\partial \mathcal{F}}{\partial V}\right)_{T}=\rho\left(\frac{\partial f_{\text {bulk }}}{\partial \rho}\right)_{T}-f_{\text {bulk }},
$$

where $\mathcal{F}$ and $f_{\text {bulk }}$ denote the free energy and bulk free energy density, respectively. Hence, the pressure tensor contains all the information regarding the local thermodynamics of the fluid and the capillary terms. In particular, the coefficient $w$ penalizes the existence of density gradients $\partial_{\alpha} \rho$ and is related to the fluid surface tension $[19,62]$.

To describe the fluid thermodynamics, we use a van der Waals fluid whose free energy density and pressure are

$$
\begin{gathered}
f_{\text {bulk }}(\rho, T)=\rho k_{B} T\left[\ln \left(\frac{\rho \Lambda^{3}}{1-\rho b}\right)-1\right]-a \rho^{2}, \\
P_{\text {bulk }}=\frac{\rho k_{B} T}{1-\rho b}-a \rho^{2} .
\end{gathered}
$$

The van der Waals parameters $a$ and $b$ and the de Broglie wavelength $\Lambda$ are set so as to represent, on the one hand, the density of liquid water at $297 \mathrm{~K}$ and atmospheric pressure and, on the other hand, the values of the critical pressure and temperature $P_{c}$ and $T_{c}$, yielding the relation

$$
\begin{aligned}
& P_{c}=\frac{a}{27 b^{2}}=22 \mathrm{MPa}, \\
& \rho_{c}=\frac{1}{3 b}=322 \mathrm{~kg} / \mathrm{m}^{3}, \\
& T_{c}=\frac{8 a}{27 b k_{B}}=647.3 \mathrm{~K},
\end{aligned}
$$

where $k_{B}$ is the Boltzmann constant. The parameter $w$ appearing in the square gradient terms in Eq. (6) is chosen to match the surface tension $\gamma=0.72 \times 10^{-3} \mathrm{~J} \mathrm{~m}^{-2}$ of water at $T=297 \mathrm{~K}$, as detailed in Ref. [19].

A word is in order regarding the value of the Clapeyron coefficient $l$, for which we have considered the dependence on temperature in addition to the dependence on density,

$$
l(\rho, T)=l_{\text {vap }}(T)+\frac{\rho(r)-\rho_{\text {vap }}}{\rho_{\text {liq }}-\rho_{\text {vap }}}\left(l_{\text {liq }}(T)-l_{\text {vap }}(T)\right),
$$

where $l_{\mathrm{liq}}(T)$ and $l_{\text {vap }}(T)$ are the temperature-dependent values of the Clapeyron coefficient at a given temperature $T$ for a liquid and vapor of density $\rho_{\text {liq }}$ and $\rho_{\text {vap }}$.

The dissipative stress tensor is

$$
D_{\alpha \beta}=\eta\left(\partial_{\alpha} v_{\beta}+\partial_{\beta} v_{\alpha}\right)+\left(\mu-\frac{2 \eta}{3}\right) \nabla \cdot \mathbf{v} \delta_{\alpha \beta} .
$$

We assume that the shear and bulk viscosities $\eta$ and $\mu$ are related by $\mu \simeq 5 \eta / 3$ [63] as is the case for hard-sphere liquids. Since the density $\rho$ in Eq. (3) is a field with large spatial variations, we need to account for the variations of the thermophysical and transport coefficients with the local density. For simplicity, we choose a linear relationship between these parameters and the density. As an example, the local shear viscosity is given by

$$
\eta(\mathbf{r})=\eta_{\text {vap }}+\frac{\rho(\mathbf{r})-\rho_{\text {vap }}}{\rho_{\text {liq }}-\rho_{\text {vap }}}\left(\eta_{\text {liq }}-\eta_{\text {vap }}\right),
$$

where the subscripts vap and liq refer to the bulk values at $297 \mathrm{~K}$. The thermophysical and transport coefficients of liquid water and vapor at $297 \mathrm{~K}$ and atmospheric pressure are summarized in Table I.

The boundary conditions at the interface between the fluid and the nanoparticle, including the continuity of pressure, the vanishing of the normal velocity, and the no-slip condition for the tangential velocity, are implemented following the scheme described in Ref. [19]. The temperature evolution of 
TABLE I. Thermophysical parameters in liquid water and in vapor at $297 \mathrm{~K}$ in SI units. $\hat{\rho}$ is the mass per unit volume, and $C_{v}$ the heat capacity per unit volume

\begin{tabular}{lcccc}
\hline \hline Phase & $\hat{\rho}\left(\mathrm{kg} / \mathrm{m}^{3}\right)$ & $C_{v}(\mathrm{~J} / \mathrm{kg} / \mathrm{K})$ & $k(\mathrm{~W} / \mathrm{m} / \mathrm{K})$ & $\eta(\mathrm{Pa} \mathrm{s})$ \\
\hline Liquid & 997.10 & $4.13 \times 10^{3}$ & 0.606 & $8.98 \times 10^{-4}$ \\
Vapor & $2.22 \times 10^{-2}$ & $1.44 \times 10^{3}$ & 0.019 & $9.99 .9 \times 10^{-6}$ \\
\hline \hline
\end{tabular}

the nanoparticle is coupled to that of the fluid through the conductive flux $\phi$ and fluid boundary conditions. Specifically, one imposes at the nanoparticle surface the condition

$$
\phi=-k\left(\frac{\partial T}{\partial r}\right)_{r=R_{\mathrm{np}}},
$$

where $T$ denotes the fluid temperature and the previous equation is estimated at the nanoparticle position $r=R_{\mathrm{np}}$. We have not taken into account the nanoparticle expansion due to its heating, as we have observed that this expansion plays a minor role in the amplitude of the emitted pressure wave and in the nanobubble dynamics. As detailed in Ref. [48], perfectly matched layers are implemented at the simulation domain boundaries to avoid any reflection of the pressure waves generated by the sudden vapor generation.

We consider a spherically symmetric system that allows us to focus only on the radial component of the fields. The nanoparticles have a radius ranging from 5 to $100 \mathrm{~nm}$. The simulation cell, which is centered on the nanoparticle, has a size $L=10$ microns, with $L=100 \mathrm{~nm}$ of perfectly matched boundary layer for $R_{\mathrm{np}}<50 \mathrm{~nm}$ and $L=390 \mathrm{~nm}$ otherwise. We use a time step of $0.2 \mathrm{fs}$ and a lattice constant of $0.07 \mathrm{~nm}$. The velocity field is calculated on a staggered grid, shifted from the main grid by half a lattice constant [48].

\section{RESULTS}

\section{A. Generation and propagation of the pressure wave}

In this section, we describe the physical processes underlying the appearance and propagation of pressure waves. When the nanoparticle is heated by laser illumination, a transient vapor nanobubble is generated, provided the fluence is above a threshold value $F_{\text {th }}$, which depends on the size and pulse duration, as shown in Fig. 2. As demonstrated in Ref. [19], the

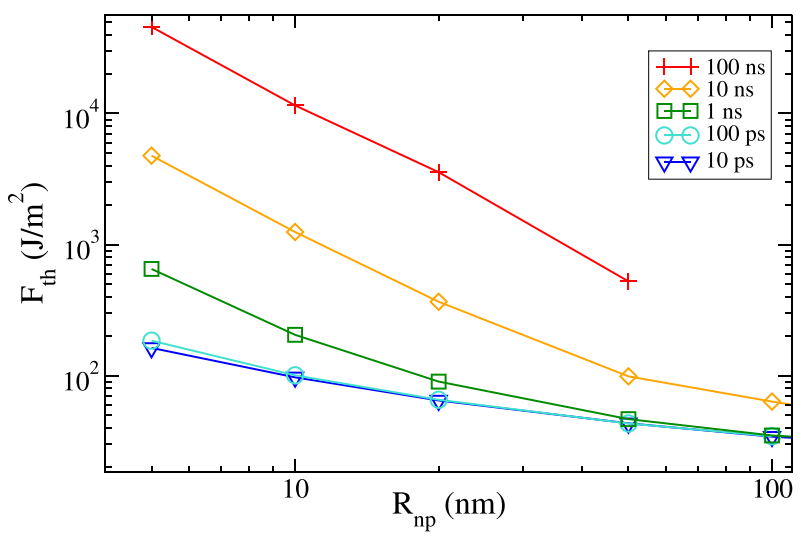

FIG. 2. Vaporization threshold fluence as a function of the nanoparticle radius, for different laser pulse durations. threshold $F_{\text {th }}$ corresponds to the crossing of the liquid/water spinodal temperature, $T_{\mathrm{sp}} \simeq 550 \mathrm{~K}$ for water, at a distance $1 \mathrm{~nm}$ from the nanoparticle surface. In the following, we focus on the pressure waves that follow vapor nanobubble generation.

As illustrated in Fig. 3, the waves are emitted at the nanoparticle surface and propagate over microns in liquid water. The waves are compressive and not tensile in nature. Their spatial extension increases during propagation, a dispersion that may be qualitatively explained by the density dependence of the compressibility induced by the high pressure levels. Nevertheless, one can infer a wave propagation speed, whose value $c_{w} \simeq 1700 \mathrm{~m} / \mathrm{s}$ is found to be above the value of the water sound velocity, in the initial fluid state prior to any heating. This relatively high value of the sound velocity may be explained by two factors. First, for the fluence levels considered here, the overpressure corresponding to the wave propagation is more than one order of magnitude higher than the initial pressure. Under these conditions, the sound velocity is the one characterizing the high-pressure fluid, which is typically $10 \%$ higher than the initial sound velocity [64]. Second, due to the large temperature gradient in the vicinity of the hot nanoparticle, the early stage of acoustic propagation does not occur under strictly isentropic conditions. Therefore, the sound velocity may differ from its isentropic counterpart.

As regards the wave amplitude, it decays spatially as $r^{-\alpha}$ with an exponent $\alpha=1.5$, which is typical of laser-induced stress wave propagation $[65,66]$. We investigate the pressure amplitude at a fixed distance $d$ from the nanoparticle surface. We choose $d=500 \mathrm{~nm}$, a typical distance in a biological cell environment. Note that in Fig. 3, the fluence level is fixed at $F=10 F_{\text {th }} \simeq 400 \mathrm{~J} / \mathrm{m}^{2}$, which is typical of experimental investigations of nanobubble-induced cell injury [3,30,67-69]. In spite of this relatively moderate fluence and even after propagating over $500 \mathrm{~nm}$, the pressure amplitude level may reach very high values, around hundreds of atmospheres.

The physical process at the origin of wave formation is scrutinized in Fig. 4. At the nanoparticle surface, a strong pressure increase is observed before the end of the laser pulse $(t=10 \mathrm{ps})$. The excess pressure starts to relax before vaporization occurs ( $t=15 \mathrm{ps})$. Inspection of the data also indicates that the pressure is maximum just before the bubble forms. The next step is a very sharp decrease in the pressure at the nanoparticle surface, due to the generation of the vapor phase and the subsequent huge decrease in the water temperature after bubble formation. This phenomenon has been observed in Ref. [48] and explained by the huge drop in the thermal conductance at the nanoparticle/fluid interface following the water phase change.

The first pressure wave is followed by a series of echoes. These secondary waves correspond to the generation of 

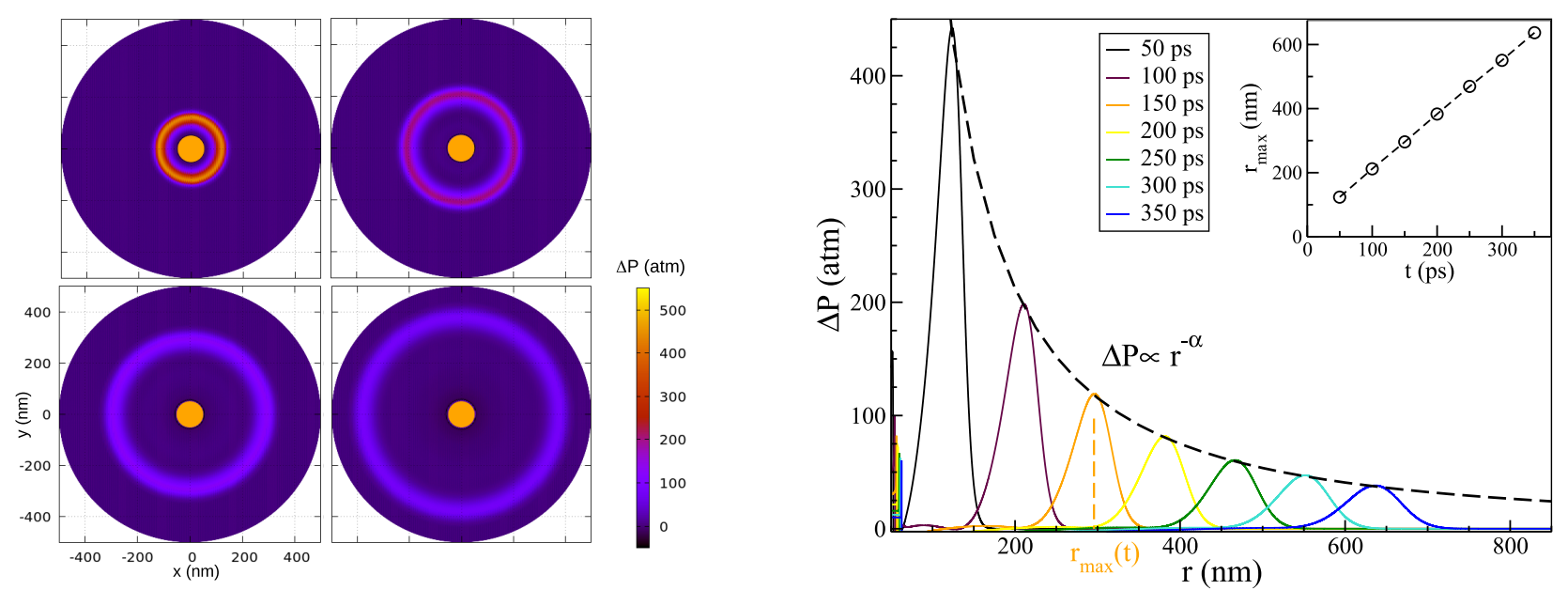

FIG. 3. Propagation of nanocavitation-induced pressure waves. Left: Two-dimensional representation of the pressure wave propagation around the nanoparticle. The excess pressure is plotted for different times, ranging from 52 to 252 ps. The nanoparticle is represented by the yellow circle at the center. Right: Propagation away from the nanoparticle of the overpressure $\Delta P=P-P_{\infty}$ after the first vaporization event. The dashed line shows the decreasing pressure amplitude while it travels away from the nanoparticle. Inset: Time evolution of the maximum wave position with time. The nanoparticle radius is $R_{\mathrm{np}}=50 \mathrm{~nm}$, the pulse duration $\tau_{\mathrm{p}}=10 \mathrm{ps}$, and the fluence $F=10 F_{\mathrm{th}}=430 \mathrm{~J} / \mathrm{m}^{2}$.

secondary bubbles following bubble collapse, as evidenced by Fig. 5. After the first bubble collapse, a second pressure wave is emitted. When the maximum radius is reached $(t \simeq$ $1000 \mathrm{ps})$, the pressure within the bubble starts to increase again. However, the vapor pressure during the collapse does not reach the levels of pressure of the acoustic wave emitted. Immediately after the first bubble collapse $(t \simeq 2800 \mathrm{ps})$, heat transfer from the nanoparticle to the liquid is reactivated, and a second vaporization event occurs, accompanied by the generation of a second pressure wave. A third vaporization event is observed at $t \simeq 4800 \mathrm{ps}$, and so on. This repeated emission of waves, reminiscent of a "hammer" effect, opens the way to the generation of repeated thermomechanical damage at a micron distance from the nanoparticle surface. Though the

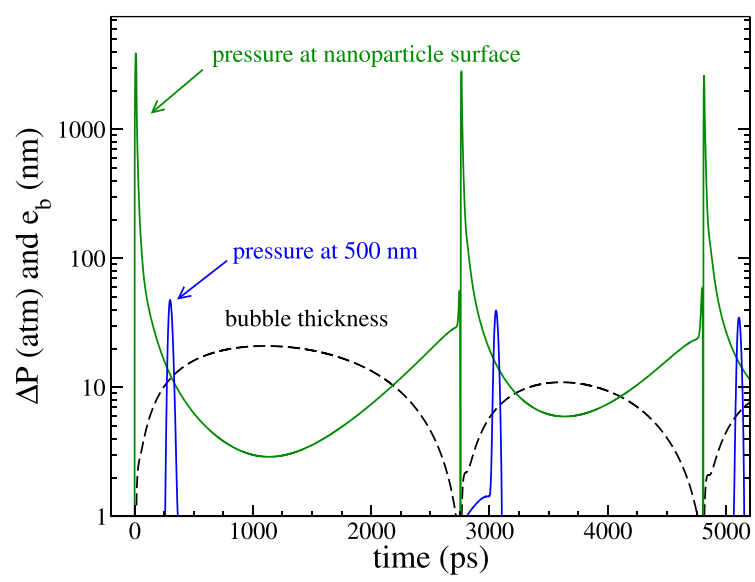

FIG. 4. Pressure at the nanoparticle surface and at a distance $d=500 \mathrm{~nm}$ from the nanoparticle surface, as a function of the time. The figure also shows the temporal evolution of the bubble thickness $e_{b}(t)=R_{b}(t)-R_{\mathrm{np}}$. The average velocity of the first bubble is $v_{b} \simeq 16 \mathrm{~m} / \mathrm{s}$. The nanoparticle radius is $R_{\mathrm{np}}=50 \mathrm{~nm}$, the pulse duration $\tau_{\mathrm{p}}=10 \mathrm{ps}$, and the fluence $F=10 F_{\mathrm{th}} \simeq 430 \mathrm{~J} / \mathrm{m}^{2}$. phenomena are similar in nature, the generation of waves is influenced by the duration of the pulse. The cases of a short pulse, $\tau_{\mathrm{p}}=10 \mathrm{ps}$, and a longer pulse, $\tau_{\mathrm{p}}=1 \mathrm{~ns}$, are shown in Fig. 5. With the short pulse, a first large bubble is inflated, which is followed by a series of smaller nanobubbles. This contrasts with the case of a long pulse, in which the first small nanobubble is followed by a series of larger secondary bubbles. Note that the amplitude of the secondary waves does not vary much with the pulse duration.

A remark is in order on the origin of the pressure wave. It is important to realize that the bubble wall velocity $\left(v_{b} \simeq\right.$ $16 \mathrm{~m} / \mathrm{s}$ ) is typically two orders of magnitude lower than the pressure wave velocity $c_{w}$. This clearly indicates that pressure waves are emitted by the explosive phase change due to fluid heating and that their propagation is not governed by the bubble dynamics. Hence, wave propagation cannot be seen as a radiation from the expanding bubble, a phenomenon which is shown here to be completely negligible.

Besides, acoustic waves do not originate in the collapse of the vapor bubble. Indeed, as shown in Fig. 4, for a shortduration pulse of fluence $10 F_{\text {th }}$, the maximal pressure at the particle surface is of the order of $3000 \mathrm{~atm}$, which is two orders of magnitude larger than the pressure during the bubble collapse (of the order of $30 \mathrm{~atm}$ ). The latter value is typical of pressure levels predicted by Rayleigh-Plesset types of models. Indeed, for short pulses, Rayleigh-Plesset models predict a peak pressure of $40 \mathrm{~atm}$ for a nanoparticle radius $R_{\mathrm{np}}=40$ $\mathrm{nm}$ and a fluence $F=1000 F_{\text {th }}[27,70]$. In the Appendix, we illustrate the huge gap between the Rayleigh-Plesset predictions and the phase-field results for the maximal emitted pressure. This huge gap may be explained qualitatively by the different physical origin of the peak pressure: in RayleighPlesset models, the peak pressure corresponds to the pressure emitted by the bubble collapse. In the phase-field simulation the maximal pressure is observed at the formation of a greatly out-of-equilibrium liquid-vapor interface. This is consistent with the conclusion that Rayleigh-Plesset types of 

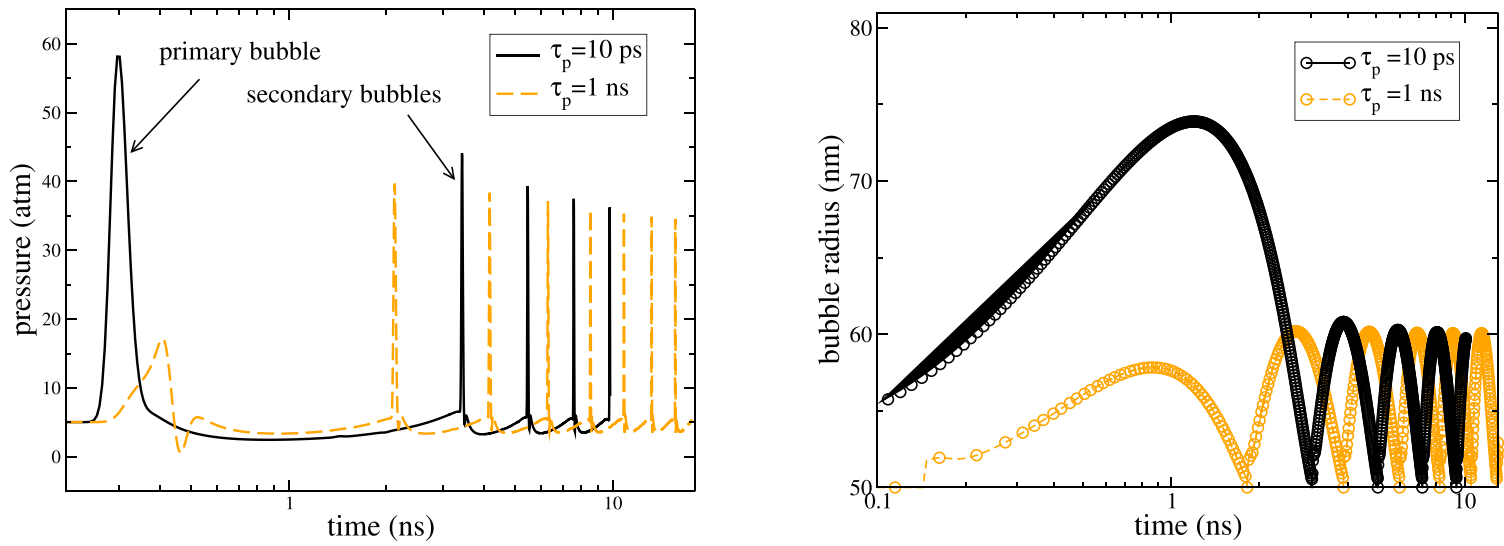

FIG. 5. Left: Time evolution of the pressure $P$ at a distance of $500 \mathrm{~nm}$ from the nanoparticle surface for two laser pulse durations, $\tau_{\mathrm{p}}=$ $10 \mathrm{ps}$ (solid lines) and $\tau_{\mathrm{p}}=1 \mathrm{~ns}$ (dashed lines). Right: Corresponding evolution of the nanobubble radius $R(t)$. The nanoparticle radius is $R_{\mathrm{np}}=50 \mathrm{~nm}$ and the fluence is $F=16 F_{\mathrm{th}} \simeq 690 \mathrm{~J} / \mathrm{m}^{2}$.

models tend to severely underpredict the intensity of acoustic emission generated by the sudden formation of a vapor nanobubble.

Another observation that is essential for the applications is that the wave rising time $\tau_{\mathrm{r}}$ is found to be short, of the order of $100 \mathrm{ps}$. This small value can be ascribed to the greatly out-of-equilibrium nature of pressure emission, which is concomitant with sudden nanobubble generation. As a consequence of this very fast relaxation, the maximal pressure at the particle surface is orders of magnitude larger than the inner bubble pressure. Therefore, models based on RayleighPlesset equations would completely fail to account for the pressure intensity emitted by the nanobubble [27]. For the particular case analyzed here corresponding to a fluence $F=$ $10 F_{\text {th }}$, the maximum pressure level at a distance of $500 \mathrm{~nm}$ is approximately $50 \mathrm{~atm}$. A second practical consequence is that the high pressure levels $P_{\max }$ combined with the short rising times $\tau_{\mathrm{r}}$ yield stress gradients that are high enough to induce significant thermomechanical damage in a biological cell environment, as we analyze in detail below.

\section{B. Influence of pulse duration and nanoparticle size}

We now investigate how the pressure emission and propagation depend on the laser pulse duration and nanoparticle size. The evolution of the pressure wave amplitude, emitted by both primary and secondary nanobubbles, as a function of the laser fluence and for different nanoparticle sizes is quantified in Fig. 6. Here again, two different behaviors are highlighted, depending on the pulse laser duration $\tau_{\mathrm{p}}$. The strongest effects are observed for the short pulse, $\tau_{\mathrm{p}}=10 \mathrm{ps}$. In this case, the pressure amplitude corresponding to the first wave increases continuously with the laser fluence when the vaporization threshold is crossed. Below the vaporization threshold $F<$ $F_{\text {th }}$, the pressure emitted is due to the fluid dilation induced by local heating. The amplitude of the secondary wave increases with the fluence above the threshold $F_{\text {th }}$ and saturates above a fluence $F \simeq 4 F_{\text {th }}$. Comparing the amplitudes of the primary and secondary waves, they have comparable amplitudes for low fluences, but for $F>6 F_{\text {th }}$ the largest excess pressure is observed for the first vaporization. Yet, secondary vaporizations still generate a series of pressure waves of relatively high amplitude [71].

In the case of a long pulse ( $\left.\tau_{\mathrm{p}}=1 \mathrm{~ns}\right)$, the pressure maximal amplitude levels off when the vaporization threshold is crossed. At low fluences, $F<F_{\text {th }}$, the pressure amplitudes are relatively low. Beyond $F_{\text {th }}$, the amplitude is somewhat higher but still lower than in the picosecond case (for a given value of the fluence). In this fluence regime, the pressure amplitude is higher for the secondary vaporizations. This implies that cumulative effects may be efficient in the case of long pulse durations. Again, the amplitude of the secondary pressure waves saturates for high fluences, $F>4 F_{\text {th }}$. Note further that the magnitude of the secondary pressure waves is also almost the same for the 1-ns and 10-ps pulse durations, when the fluence is fixed at $F=500 \mathrm{~J} / \mathrm{m}^{2}$.

The insets in Fig. 6 analyze the particle size dependence of primary and secondary waves, for a fluence $F=10 F_{\text {th }}$. Again, two behaviors may be distinguished, depending on the laser pulse duration. When $\tau_{\mathrm{p}}=10 \mathrm{ps}$, the first vaporization gives the largest pressure, while secondary vaporizations are of smaller yet comparable amplitudes. Overall, the amplitudes of both primary and secondary waves increase with the particle size. For $\tau_{\mathrm{p}}=1 \mathrm{~ns}$, the amplitudes of both primary and secondary vaporizations increase with the nanoparticle size. However, in contrast to the short pulse duration, the amplitudes of the secondary waves are stronger than those of the primary waves. Again, cumulative effects may be particularly efficient in this regime, as nanobubble rebounds will give rise to repeated high-amplitude pressure waves.

A remark is in order regarding the possible effect of dissolved gas. All our simulation results concern a situation where both the first and the secondary bubbles are made of pure vapor. It is legitimate to wonder what the influence of dissolved gas would be. Indeed, as demonstrated experimentally $[15,16]$, nanobubble generation in the presence of uncondensable gas occurs in two steps: the first generated nanobubble is made of pure vapor; the secondary bubbles may be enriched by dissolved gas. However, this process occurs after a crossover time corresponding to the diffusion time of 

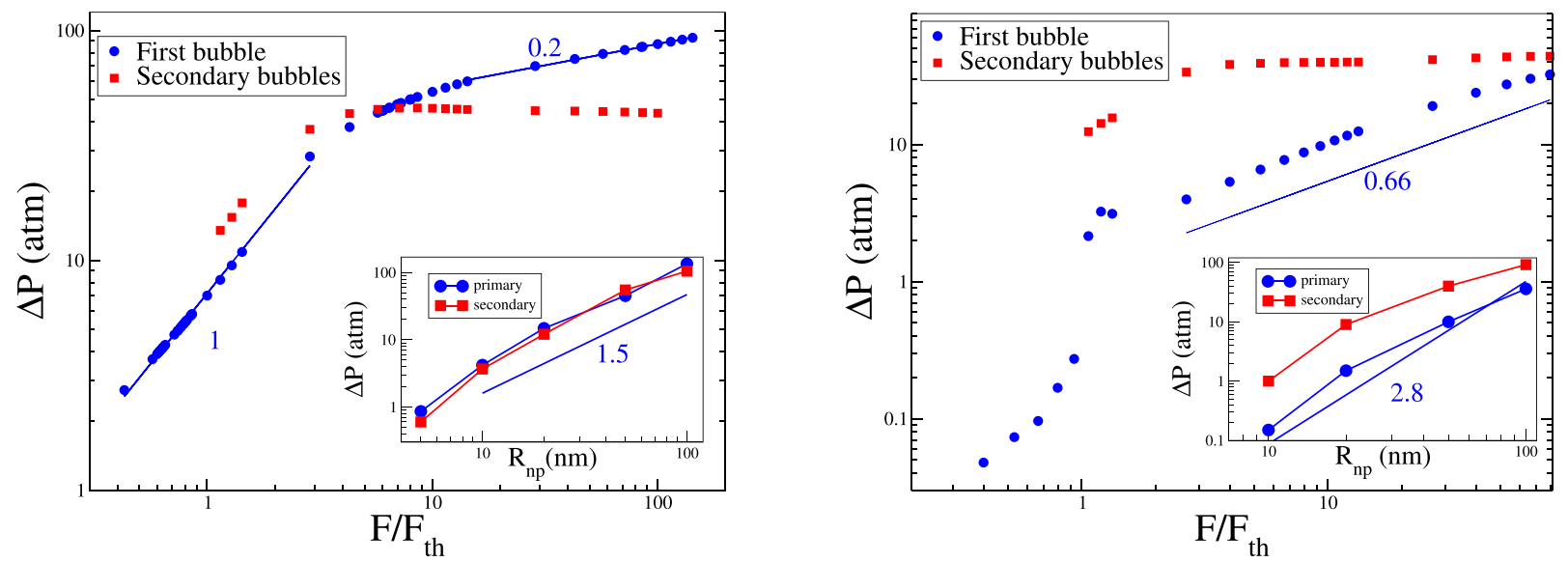

FIG. 6. Maximum pressure increase at a distance of $500 \mathrm{~nm}$ from the nanoparticle surface as a function of the laser fluence $F$. Blue circles refer to the first vaporization. Red squares correspond to the maximum pressure increase observed among all the secondary vaporizations. The threshold fluence here is $F_{\mathrm{th}}=43 \mathrm{~J} / \mathrm{m}^{2}$ and the nanoparticle radius $R_{\mathrm{np}}=50 \mathrm{~nm}$. Insets: Maximum pressure as a function of the nanoparticle size $R_{\mathrm{np}}$ for a fixed fluence, $F=500 \mathrm{~J} / \mathrm{m}^{2}$. Two pulse durations are considered: $\tau_{\mathrm{p}}=10 \mathrm{ps}$ (left) and $\tau_{\mathrm{p}}=1 \mathrm{~ns}$ (right).

the dissolved gas. This time is typically in the microsecond range [16]. Therefore, we conclude that before this crossover time, all our results obtained in the absence of dissolved gas should hold. Only after a long crossover time, which is well beyond our investigated time, might the presence of dissolved gas affect our predictions.

\section{Thermodynamic evolution}

To better interpret the different behaviors observed so far, Fig. 7 displays the evolution of the thermodynamic state of the fluid at the particle surface for two pulse durations, in a pressure-density $(P-\rho)$ diagram. The general thermodynamic evolution is similar in all the cases analyzed: the liquid prior to any heating is at the saturation value. After nanoparticle excitation, the fluid is locally strongly heated on a very short time scale. After reaching their maximal values, the pressure and temperature suddenly drop before the local density $\rho$ becomes smaller than the fluid critical density $\rho_{c}$ and the first nanobubble is generated. The nanobubble grows until the local

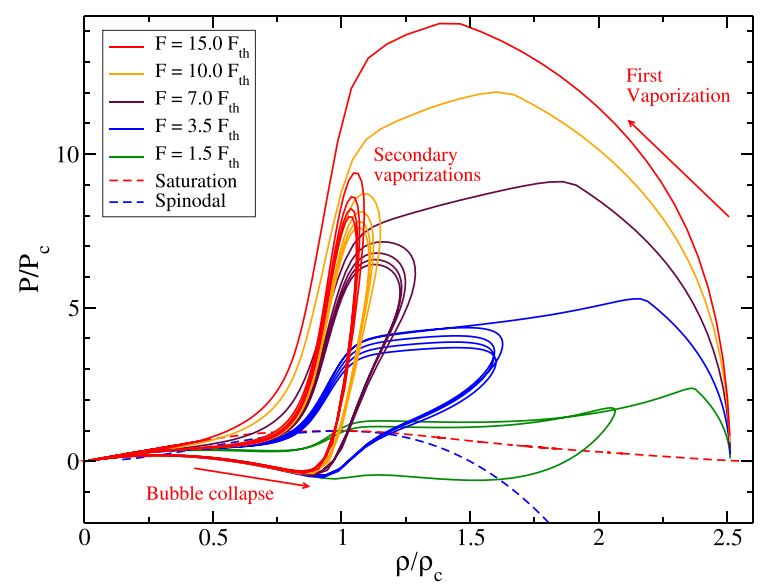

density reaches a minimal density, after which the bubble collapses. As the nanobubble acts as a vapor blanket which thermally insulates the nanoparticle from the fluid, the liquid may see a very hot nanoparticle when the bubble collapses, the latter step occurring under quasi-isothermal conditions, as already shown [48]. If the fluence is high enough, a second nanobubble is generated. This nanobubble follows another thermodynamic path, and Fig. 7 shows that its evolution is characterized by loops in the pressure-density phase diagram.

The thermodynamic path depends strongly on the pulse duration $\tau_{\mathrm{p}}$. For the fastest pulse, $\tau_{\mathrm{p}}=10 \mathrm{ps}$, the fluid pressure reaches a first maximum well above the water critical pressure $P_{c}$ before decreasing slowly until nanobubble generation. The maximal pressure levels are always higher for the first vaporization event than for the secondary ones. This conclusion is in line with the observation that, for short pulse durations, primary waves yield the strongest pressure wave amplitudes. By contrast, for the longer pulses analyzed, the maximal pressure levels attained before the first vaporization are significantly lower than in the fast pulse case. Consequently, for a constant

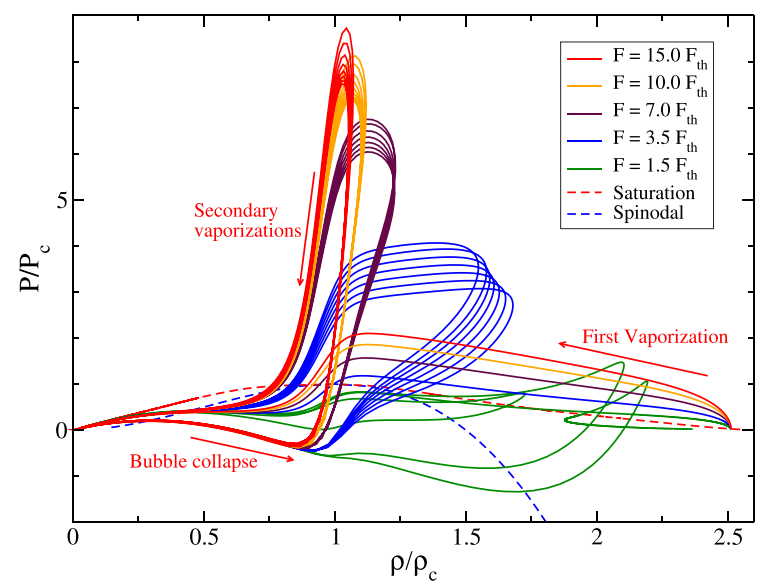

FIG. 7. Evolution of the thermodynamic state of the fluid at the particle surface in a pressure-density diagram. We consider here $R_{\mathrm{np}}=$ $50 \mathrm{~nm}$ and two pulse durations: $\tau_{\mathrm{p}}=10 \mathrm{ps}$ (left) and $\tau_{\mathrm{p}}=1 \mathrm{~ns}$ (right). Here $P_{c}=217 \mathrm{~atm}$ and $\rho_{c}=322 \mathrm{~kg} / \mathrm{m}^{3}$ are the critical water pressure and mass density, respectively. The threshold fluence is here $F_{\mathrm{th}}=43 \mathrm{~J} / \mathrm{m}^{2}$. 
value of the fluence, the pressure emitted after the first vaporization event is considerably lower in the case of long-duration pulses. The secondary vaporizations generate pressure levels much higher than the first one, which is again consistent with the conclusions reached upon analyzing Fig. 6.

Overall, the analysis of the phase diagram allows us to conclude that in order to produce high-amplitude waves, the fluid should be brought locally to its supercritical state. This may be realized either for the primary bubbles, by working with ultrashort pulses, or for the secondary waves, in all cases, if we rely on cumulative effects created by acoustic echoes.

\section{Analytical model for the pressure amplitude}

In this section, we devise a simple analytical model to interpret the evolution of the pressure amplitude, as plotted in Fig. 6, as a function of the fluence $F$ and nanoparticle size $R_{\mathrm{np}}$. Consistent with the analysis of the phase diagrams displayed in Fig. 7, we consider separately the cases of shortand long-duration pulses.

\section{Short pulses}

When the laser pulse is $\tau_{\mathrm{p}}=10 \mathrm{ps}$, the fluid in the vicinity of the nanoparticle is rapidly heated up to a temperature $T_{\max }(F)$. Once this temperature is reached, the fluid state follows an adiabatic evolution down to the state point where the density matches the critical density $\rho_{c}$. At this moment, the thermal interface conductance drops, the fluid cools down, and a vapor layer is generated. The maximal pressure, which is the value of the pressure emitted just before nanobubble generation, may be approximated as

$$
\Delta P_{s} \simeq P^{\prime}\left(T_{\max }-T_{0}\right),
$$

where $P^{\prime} \equiv\left(\frac{\partial P}{\partial T}\right)_{\rho_{0}}, T_{\max }$ is the maximal temperature at the fluid surface, and $\rho_{0}$ denotes the initial fluid density prior to any heating. As given in the Supplemental Material Appendix B, an approximation for $T_{\max }$ is available when (i) the heating is assumed infinitely fast, and (ii) the dimensionless number $C_{\mathrm{np}} R_{\mathrm{np}} / C l_{K}$, where $C$ is the fluid specific heat and $l_{K}$ is the Kapitza length of the nanoparticle/water interface [72], the thermal conductivity of the fluid, the Kapitza length $l_{K}=k / 3 G$, takes values small compared to unity, which is roughly the case here. Under these conditions, the maximal temperature increase $\Delta T_{\max }=T_{\max }-T_{0}$ is

$$
\Delta T_{\max } \simeq C_{\mathrm{num}} \sqrt{\frac{G R_{\mathrm{np}}}{C_{\mathrm{np}} C k}} \zeta F \sim F R_{\mathrm{np}}^{1 / 2} .
$$

Here, $C_{\text {num }}=\sqrt{3} F_{\max }^{D} /\left(2 \pi^{3 / 2}\right) \simeq 0.084$ is a numerical constant, with $F_{\max }^{D}$ the maximum of the Dawson function. For notational convenience, we introduce $\zeta=\sigma_{\mathrm{abs}} / R_{\mathrm{np}}^{3}$, which for nanoparticles that are not too large $\left(R_{\mathrm{np}} \leqslant 50 \mathrm{~nm}\right)$ is a constant independent of the radius. The pressure amplitude at a distance $d$ from the nanoparticle follows

$$
\Delta P(d)=\Delta P_{s}\left(\frac{R_{\mathrm{np}}}{R_{\mathrm{np}}+d}\right)^{\alpha} \simeq \Delta P_{s}\left(\frac{R_{\mathrm{np}}}{d}\right)^{\alpha},
$$

where $\alpha=3 / 2$ is the exponent describing the spatial propagation of the pressure wave (see Fig. 3) and we have assumed $R_{\mathrm{np}} \ll d$. Putting everything together and neglecting the prefactor, the expression of the pressure amplitude at a distance $d$ is

$$
\Delta P(d) \simeq P^{\prime} \sqrt{\frac{G R_{\mathrm{np}}}{C_{\mathrm{np}} C k}} \zeta F\left(\frac{R_{\mathrm{np}}}{d}\right)^{3 / 2} \sim F R_{\mathrm{np}}^{2} .
$$

The regime where $\Delta P(d) \sim F$ is clearly visible in Fig. 6. It will hold until the fluid density in the vicinity of the particle, $\rho_{\max }(F)$, approaches the critical fluid density $\rho_{c}$. When this is the case, the thermal conductance at the nanoparticle/water interface drops down by orders of magnitude [48], yielding quasi-instantaneous fluid cooling. This ultrafast temperature drop is accompanied by the emission of a pressure wave, and the value of the pressure emitted is close to the fluid pressure at the density $\rho_{c}$ and temperature $T_{\max }$. This defines a critical value of the fluence $F_{c}$ such that $\rho_{\min }\left(F_{c}\right)=\rho_{c}$. Beyond $F_{c}$, the maximal fluid temperature at the surface saturates at a value such that

$$
\Delta T_{\max }^{c}=\frac{\zeta F_{c}}{C_{\mathrm{np}}},
$$

where, for $F>F_{c}$, vapor bubble generation coincides with the crossing of $T_{\max }^{c}$ at the nanoparticle surface. In this highfluence regime, the pressure amplitude at a distance $d$ from the nanoparticle surface is

$$
\Delta P(d)=P^{\prime} \Delta T_{c}\left(\frac{R_{\mathrm{np}}}{d}\right)^{3 / 2} \sim F^{0} R_{\mathrm{np}}^{3 / 2} .
$$

This is consistent with the weak dependence of the pressure amplitude shown in Fig. 6, for short pulses $\tau_{\mathrm{p}}$ and high fluences $F$. Furthermore, the scaling $\Delta P \sim R_{\mathrm{np}}^{3 / 2}$ is completely consistent with what is shown in the inset in Fig. 6. This scaling mirrors the fact that, in this high-fluence regime, pressure emission is a local process, which is independent of the nanoparticle size.

\section{Long pulses}

Let us now consider the long-pulse regime, as illustrated by the $\tau_{\mathrm{p}}=1 \mathrm{~ns}$ case in Fig. 6 . In the latter situation, the pulse duration $\tau_{\mathrm{p}}$ is longer than the vaporization time $t_{\mathrm{vap}}$, the time necessary for a nanobubble to be generated. As a consequence, only a fraction $t_{\mathrm{vap}} / \tau_{\mathrm{p}}$ of the incoming fluence $F$ serves to vaporize the fluid. To estimate the maximal pressure in the vicinity of the nanoparticle, we first use energy conservation to write

$$
\frac{t_{\mathrm{vap}}}{\tau_{\mathrm{p}}} F \sigma_{\mathrm{np}}=C \Delta \bar{T} \frac{4 \pi}{3}\left(\left(R_{\mathrm{np}}+\sqrt{D t_{\mathrm{vap}}}\right)^{3}-R_{\mathrm{np}}^{3}\right) .
$$

Here it was assumed that all the energy received serves to heat the liquid in a spherical shell of thickness $\sqrt{D t_{\text {vap }}}$, up to an average temperature $T_{0}+\Delta \bar{T}$. The equality is written at time $t_{\mathrm{vap}}$, where the surface temperature reaches the water spinodal temperature $T_{\mathrm{sp}}$. Assuming that the thickness remains small compared to the particle radius, one can obtain $\Delta \bar{T}$ and deduce a surface pressure

$$
\Delta P_{s} \simeq P^{\prime} \frac{\sqrt{t_{\mathrm{vap}}} \zeta}{4 \pi C \tau_{\mathrm{p}} \sqrt{D}} F R_{\mathrm{np}} .
$$

Now, the vaporization time $t_{\mathrm{vap}}$ may be obtained by considering the short-time behavior of the fluid surface temperature, 

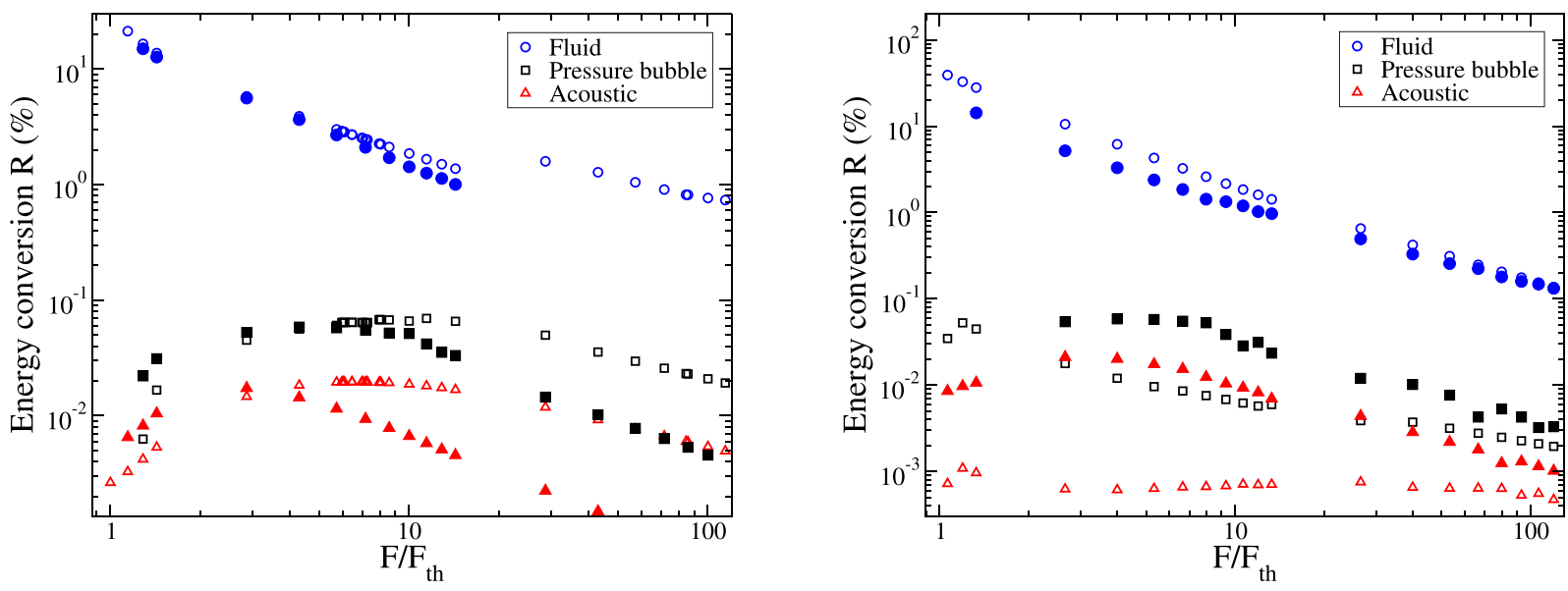

FIG. 8. Energy conversion involved in the growth of the bubble and acoustic energy carried by the pressure wave emitted from the nanoparticle surface as a function of the laser fluence $F$. Open symbols correspond to primary bubbles-waves, while filled symbols show secondary bubbles-waves. The pulse durations are $\tau_{\mathrm{p}}=10 \mathrm{ps}$ (left) and $\tau_{\mathrm{p}}=1 \mathrm{~ns}$ (right). The nanoparticle radius is $R_{\mathrm{np}}=50 \mathrm{~nm}$, and the threshold fluence is $F_{\text {th }}=43 \mathrm{~J} / \mathrm{m}^{2}$.

as given in Appendix B,

$$
t_{\text {vap }}=\frac{\pi}{3^{2 / 3}}\left(\frac{C_{\mathrm{np}}^{4} C k \Delta T_{s}^{2}}{G^{4} \zeta^{2}}\right)^{1 / 3} F^{-2 / 3} R_{\mathrm{np}}^{2 / 3},
$$

with $\Delta T_{s}=T_{\mathrm{sp}}-T_{0}$. One finally obtains the scaling for the pressure amplitude at a distance $d$ :

$$
\Delta P(d) \simeq \Delta P_{s}\left(\frac{R_{\mathrm{np}}}{d}\right)^{3 / 2} \sim F^{2 / 3} R_{\mathrm{np}}^{17 / 6} .
$$

Compared to the numerical data in Fig. 6, these scaling laws capture the main trends well.

\section{E. Energy conversion}

Finally, we quantify the energy conversion associated with the acoustic emission. Our reference is the total energy brought by the laser $E_{\mathrm{L}}=\sigma_{\mathrm{abs}} F$, where $\sigma_{\mathrm{abs}}$ is the nanoparticle absorption cross section and $F$ the laser fluence. Part of this energy is stored in the nanoparticle, and only a fraction of this energy is delivered to the fluid during bubble growth. We call $E_{\mathrm{f}}(n)$ the energy which is transmitted to the fluid during the lifetime of the $n$th bubble, which is between the collapse of the $(n-1)$ th bubble and the collapse of the $n$th bubble. It is important to realize that not all the energy transferred to the fluid $E_{\mathrm{f}}(n)$ is used for the growth of the $n$th bubble. Indeed, part of this energy is dissipated as heat in water and is therefore not employed for bubble growth. To disentangle the latter energy channel, we have defined $E_{\gamma}(n)$ as the energy effectively converted to mechanical energy. Finally, along with bubble growth, a fraction of the energy transferred to the fluid is converted to acoustic energy, which we denote $E_{\mathrm{a}}(n)$. The expressions of $E_{\mathrm{f}}(n), E_{\gamma}(n)$, and $E_{\mathrm{a}}(n)$ are given in the Appendix A.

Figure 8 shows the relative energy conversion channels, namely, fluid $E_{\mathrm{f}}(n) / E_{\mathrm{L}}$, pressure bubble $E_{\gamma}(n) / E_{\mathrm{L}}$, and acoustic $E_{\mathrm{a}}(n) / E_{\mathrm{L}}$ for the first generated bubble $n=1$ and second bubble $n=2$. First, we note that most of the energy brought by the laser is stored in the nanoparticle and not transferred to the fluid. Second, only a small fraction of this energy is converted to mechanical energy for pressure growth. The remaining energy is hence lost in water through thermal diffusion. Third, only a tiny fraction of the energy is converted to acoustic energy. The magnitude of conversion is found to be consistent with what has been reported for explosive boiling induced by microheaters [11] and previous estimates of the acoustic energy conversion around photothermal nanoparticles [37].

The existence of an optimum for the energy conversion (laser energy into mechanical energy) may be understood following the same lines as in Refs. [6] and [19]. When the laser fluence $F$ is close to the threshold $F_{\text {th }}$, the vaporization times are very long. In this regime, a significant fraction of the energy transferred to the fluid is dissipated as heat, and the energy conversion of mechanical energy is poor. By opposition, for large fluences the vaporization time becomes very short, and in this regime most of the energy supplied by the laser is stored in the nanoparticle. This explains why the relative energy to the fluid is a decreasing function of the fluence. Between these two regimes, there is an optimum where the vaporization times take intermediate values, for which the energy transferred to the fluid is not small and the heat losses by thermal diffusion are miminized. Close to this optimum in fluence, the relative energy effectively used for bubble growth is maximal.

\section{APPLICATION: MECHANISM OF DAMAGE TO A CELL MEMBRANE}

As a direct application of our results, we discuss here the possible thermomechanical effects induced by nanobubble generation on the destruction or breaching of cell membranes at the subcellular level, as illustrated in Fig. 9. Two mechanisms have been put forward to explain cancer cell destruction following nanovaporization around nanoparticles, namely, nanobubble expansion and pressure wave propagation [32]. In the first mechanism, nanobubble expansion generates strain inside the cell, and if the cell membrane is deformed by a strain high enough, it will be destroyed. For most eukaryotic 


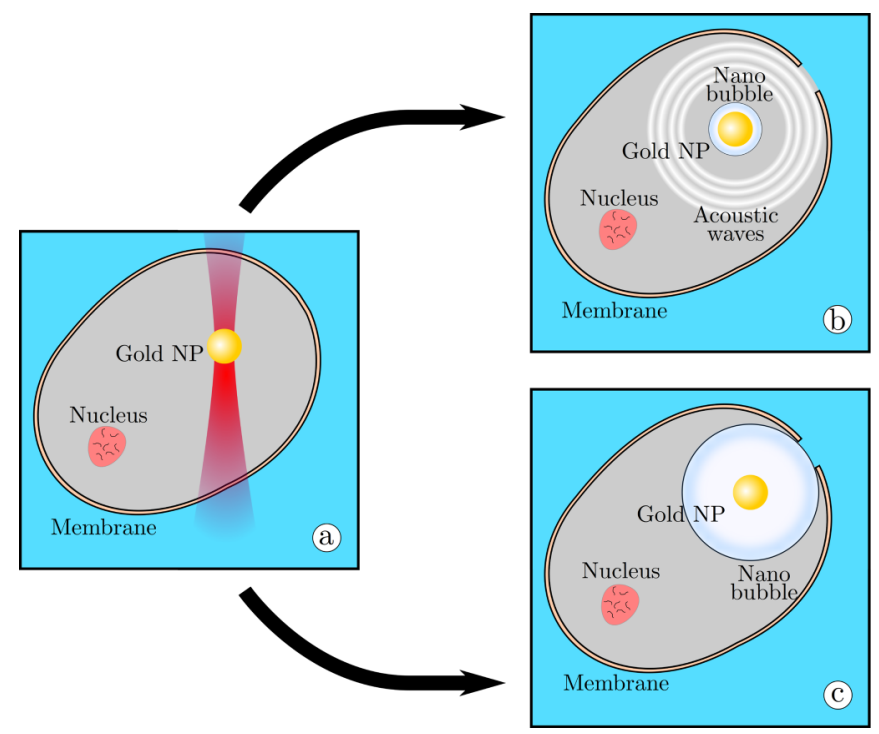

FIG. 9. Illustration of the two possible mechanisms through which nanobubble generation may lead to cell membrane disruption perforation (a): acoustic-mediated perforation (b) and nanobubble expansion and strain-induced disruption (c).

cells, the critical value of the strain is found to be close to $\epsilon_{\mathrm{cr}} \simeq 3 \%$ [73]. As regards acoustic emission, prior in vitro studies $[74,75]$ concluded that the control parameter is not the peak pressure but the pressure growth rate (or pressure rate of change) [76],

$$
\sigma=\frac{P_{\max }}{\tau_{\mathrm{r}}}
$$

where $P_{\max }$ is the maximum in pressure and $\tau_{\mathrm{r}}$ the rising time to reach it. Typically, a growth rate higher than $\sigma_{\mathrm{cr}}=50 \mathrm{~atm} / \mathrm{ns}$ leads to cell membrane alteration [75]. Based on our simulation results, we can estimate the possible damage created by an illuminated nanoparticle as a function of the nanoparticle size, laser fluence, and pulse

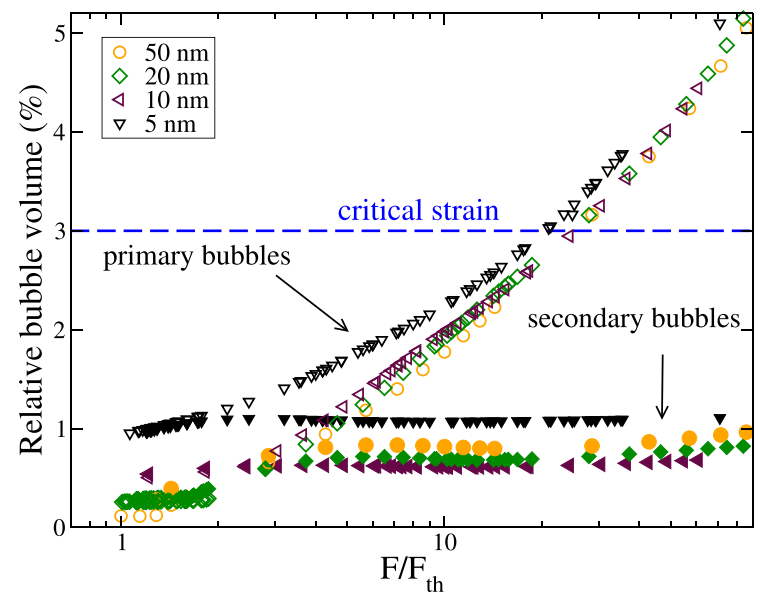

duration. We concentrate on a fluence interval $F$ in the range $[100,5000] \mathrm{J} / \mathrm{m}^{2} \simeq\left[F_{\mathrm{th}} ; 50 F_{\mathrm{th}}\right]$, corresponding to fluence levels typical of experiments [3,30,67-69]. To quantify possible damage and decide which of the two above-mentioned mechanisms is responsible for membrane disruption, we adopt a simplified model, where the irradiated nanoparticle is at the center of a cell of radius $R_{c}=500 \mathrm{~nm}$.

Consider first the effect of nanobubble expansion. Given our simplified model, the strain is simply equal to the relative bubble expansion and its maximal value is $\Delta V_{\max } / V=$ $\left(\left(e_{\max }+R_{\mathrm{np}}\right)^{3}-R_{\mathrm{np}}^{3}\right) / R_{\mathrm{np}}^{3}$, where $e_{\max }$ is the maximal vapor bubble thickness. As shown in Fig. 10, the maximal bubble expansions $\Delta V_{\max } / V$ depend only weakly on the nanoparticle size. They remain around $1 \%$ for all the secondary generated bubbles, while for the primary bubbles, they do no exceed $2 \%$ in the fluence range $\left[F_{\mathrm{th}} ; 10 F_{\mathrm{th}}\right]$. We conclude that membrane destruction due to bubble expansion is quite unlikely below $10 F_{\text {th }}$. Only with larger fluences, $F>20 F_{\text {th }}$, would the maximal relative deformation reach the critical value $\epsilon_{\mathrm{cr}} \simeq 3 \%$. In all cases, membrane destruction is only possible for short pulse durations and is triggered by the first bubble generated.

Let us discuss now the effect of the emitted acoustic wave. Shown in Fig. 10 is the growth rate $\sigma$ as a function of the laser fluence. For almost all nanoparticle sizes and fluences above threshold, it is above the critical value $\sigma_{\mathrm{cr}}=50 \mathrm{~atm} / \mathrm{ns}$. Therefore, we conclude that the interaction between the pressure wave and the cell membrane is the most likely cause of cell damage, at least when the fluence is lower than $1000 \mathrm{~J} / \mathrm{m}^{2}$. Nanobubble expansion may cause cell membrane disruption only for higher fluence levels.

It is thus clear that in our idealized situation, acoustic propagation has a more drastic effect than nanobubble expansion. This being said, several remarks are in order. (i) Our simplified model concerned nanobubble generation around a single nanoparticle supposed to be located in the middle of the cell. Clearly, the possible damage induced by either mechanism will depend on the precise location of the nanoparticle, and more pronounced effects are to be predicted for nanoparticles in the vicinity of the cell membrane or in the situation where

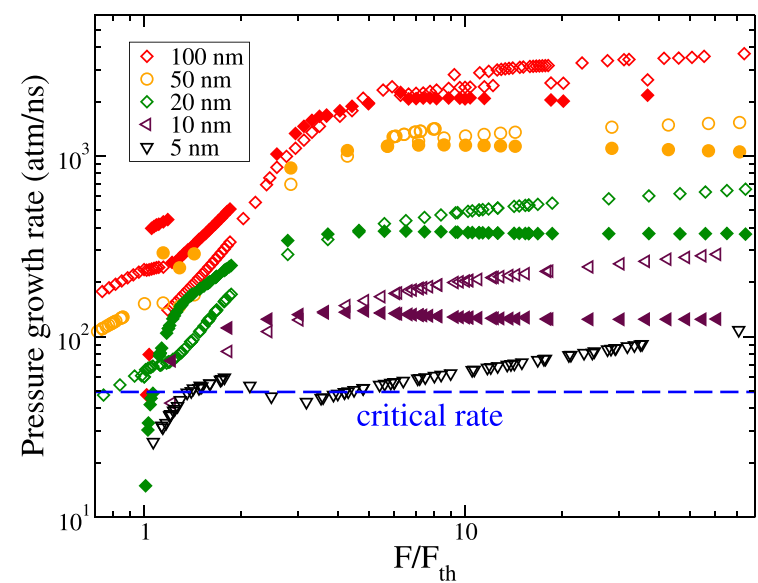

FIG. 10. Left: Maximum relative volume of expanding bubbles, as a function of the laser fluence $F$. Open and filled symbols correspond to the first and secondary nanobubbles, respectively. Right: Pressure growth rate, as defined by Eq. (25), calculated at a distance of $500 \mathrm{~nm}$ from the nanoparticle surface, as a function of the laser fluence $F$ normalized by the bubble fluence threshold $F_{\text {th }}$. The values of the size-dependent fluence threshold $F_{\text {th }}$ are given in Fig. 2. In both cases, the dashed lines indicate the typical critical value of strain leading to membrane disruption. The pulse duration here is $\tau_{\mathrm{p}}=10 \mathrm{ps}$. 
the nanoparticles are inseminated by endocytosis, leading to the mechanical injury of the vesicle. (ii) In a real situation, not one but many nanoparticles are inseminated in a diseased cell. Given that nanobubble generation is a deterministic processcontrary to nucleation - the acoustic waves created by the different nanoparticles will interfere coherently and the mechanical effect of the acoustic waves will be additive. Also, the strain levels seen by the membrane will increase with the number of irradiated nanoparticles. We leave an exhaustive study of all these effects to a future study. Our discussion around Fig. 10 was aimed at balancing the effect of nanobubble expansion and acoustic emission in a idealized situation, and as such we predict relatively low fluence bounds for cell injury. In a more realistic situation, collective effects will play a role but we anticipate that our conclusions will hold in this case too. (iii) Finally, we discuss the relevance of another mechanism, as suggested by Volkov et al. [26]. The acoustic wave that we characterized has a purely compressive nature but it could become tensile upon reflection by organelles floating in the cytosol. However, given the typical reflection coefficient of the organelles ( $\mathcal{R} \simeq 0.05$ as estimated in Ref. [26]) and the pressure peaks of the compressive waves $\left(P_{\max } \simeq 100 \mathrm{~atm}\right.$ for $F \simeq 5000 \mathrm{~J} / \mathrm{m}^{2}$ ), this mechanism is quite unlikely to alter the cell integrity.

\section{CONCLUSION}

To summarize, we have investigated numerically the process of pressure emission accompanying vapor nanobubble generation around gold nanoparticles illuminated by picosecond or nanosecond laser pulses close to their plasmon resonance frequency. As the surrounding water undergoes ultrafast vaporization, strong pressure waves are emitted that propagate in the liquid water. A recurrent observation is that the processes involved - in particular, the formation of a vapor nanobubble-are strongly out of equilibrium.

There are two important consequences. The first is that the peak pressure emitted from the nanoparticle surface is rather high, above the water critical pressure $P_{c}=220 \mathrm{~atm}$. This reflects the spinodal nature of the vapor generation process. To maximize the peak pressure, it is clear that the fluid should be brought to its supercritical state, which may be achieved either with primary bubbles, working with ultrashort pulses, or with secondary bubbles, thanks to the nanobubble rebounds. The second consequence is that the pressure rising time is short, of the order of $100 \mathrm{ps}$. Taken together, these two features determine the possible damage that plasmonic nanobubbles may cause in a biological cell environment. The combination of a high peak pressure and a short rising time leads to pressure gradients that are sufficiently strong to destroy the cell membrane. Importantly, this conclusion holds even for fluence levels close to the nanobubble fluence threshold. In contrast, the relative values of strains generated by nanobubble expansion turn out to be too small to induce any noticeable mechanical damage, even for relatively high fluence levels. It is only with fluences exceeding the threshold by several orders of magnitude that the strain would become appreciable.

We have addressed here an idealized situation where the irradiated nanoparticle is located in a model biological cell. The possible mechanical damage was assessed based on the damage created by a single acoustic wave. However, it is clear that in all situations analyzed, a series of equal-amplitude waves should impinge on the membrane cell. Importantly, the amplitudes of these echoes are only weakly damped as a result of weak viscous dissipation. Hence, it should be possible to realize membrane breaching with repeated action of moderate-amplitude nanobubble-generated pressure waves. The microscopic mechanisms underlying these effects remain partly to be explored. A direction for future work is thus to characterize the effects of repeated acoustic waves that emerge from the nanobubble rebounds. Combining our modeling approach with molecular dynamics simulations will open the way to a systematic and quantitative investigation of these effects.

\section{ACKNOWLEDGMENTS}

We are grateful to C. Loison, T. Dehoux, A. Chemin, D. Amans, and F. Amblard for interesting discussions. Part of the computational work herein was carried out on the PSMN Supercomputer at the Centre Blaise Pascal, ENS Lyon (France).

\section{APPENDIX A}

\section{Comparison between phase-field simulations and Rayleigh-Plesset predictions}

In this Appendix, we perform a quantitative comparison between the phase-field model and the predictions of the Rayleigh-Plesset equation. The Rayleigh-Plesset predictions are taken from Ref. [27]. For the sake of comparison, we consider here both a short pulse duration, $\tau_{\mathrm{p}}=10 \mathrm{ps}$, and a long pulse duration, $\tau_{\mathrm{p}}=10 \mathrm{~ns}$, while the fluence levels range from $F=0.1 F_{\text {th }}$ to $F=1000 F_{\text {th }}$. Figure 11 displays the evolution of the maximal pressure amplitude as a function of the fluence, for different nanoparticle sizes. As shown, the phase-field simulations predict maximal pressure amplitudes that may be orders of magnitude higher than those with the Rayleigh-Plesset model. These data suggest that RayleighPlesset types of models cannot capture the physics of acoustic emission, which is essentially an out-of-equilibrium effect.

\section{Energy conversion parameters}

To quantify the relative part of the energy that is transmitted to the fluid, it is useful to introduce the following quantities:

$$
\begin{gathered}
\Delta T_{\mathrm{np}}^{*}(t)=E_{\mathrm{L}} / V_{\mathrm{np}} C_{\mathrm{np}} \times \begin{cases}1 & \text { if } t \geqslant \tau_{\mathrm{p}}, \\
t / \tau_{\mathrm{p}} & \text { otherwise, }\end{cases} \\
E_{\mathrm{f}}(1)=V_{\mathrm{np}} C_{\mathrm{np}}\left[\Delta T_{\mathrm{np}}^{*}(t)-\Delta T_{\mathrm{np}}(1 \mathrm{st} \text { vap })\right], \\
E_{\mathrm{f}}(n)=E_{\mathrm{f}}(n-1)+V_{\mathrm{np}} C_{\mathrm{np}}\left[\Delta T_{\mathrm{np}}^{*}(t)-\Delta T_{\mathrm{np}}(n \text {th vap })\right] .
\end{gathered}
$$

$E_{\mathrm{L}}$ is the energy brought by the laser and $\Delta T_{\mathrm{np}}^{*}$ is the increase in nanoparticle temperature if all the energy brought by the laser is stored in the nanoparticle. Equation (A2) is the energy provided to the fluid before the first vaporization (between $t=$ 0 and $t=t_{\text {vap }}$ ). Equation (A3) is the energy provided to the 

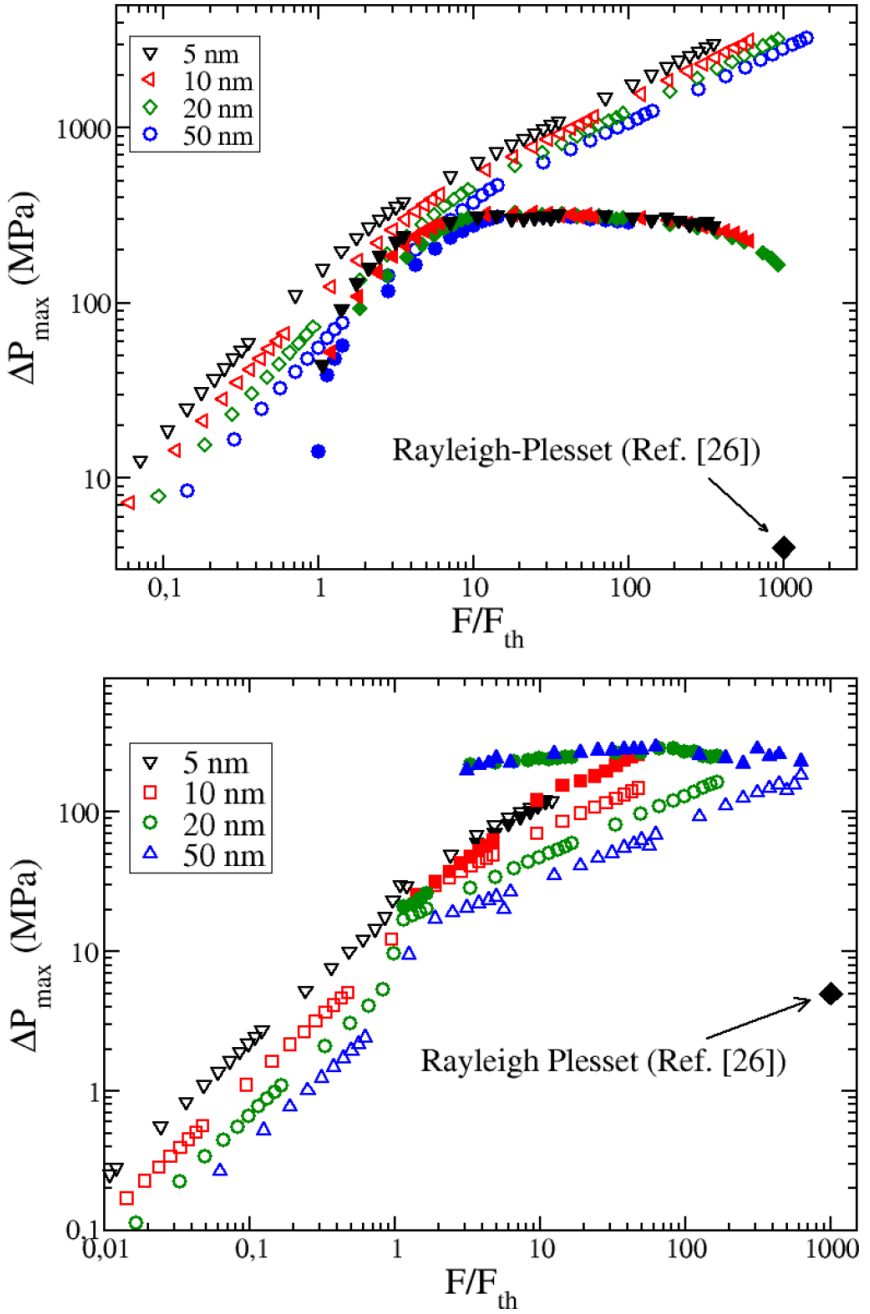

FIG. 11. Maximal pressure amplitude at the nanoparticle surface as a function of the fluence $F$ and for different nanoparticle sizes: $R_{\mathrm{np}}=5,10,20$, and $50 \mathrm{~nm}$. Values of the size-dependent fluence threshold are given in Fig. 2. Top: The laser pulse duration is $\tau_{\mathrm{p}}=$ 10 ps. Bottom: $\tau_{\mathrm{p}}=10 \mathrm{~ns}$. Open and filled symbols correspond to primary and secondary bubbles, respectively. For the sake of comparison, we have also represented the Rayleigh-Plesset prediction from Ref. [27] corresponding to the same pulse durations and a nanoparticle radius $R_{\mathrm{np}}=40 \mathrm{~nm}$.

fluid for the $n$th vaporization, which is between the collapse of the $(n-1)$ th bubble and the generation of the $n$th bubble. The energy stored during the growth of the $n$th bubble is

$$
E_{\gamma}(n)=4 \pi \int_{t_{\text {vap }}(n)}^{t_{\max }(n)} 2 \gamma R_{b} \dot{R}_{b} d t,
$$

where $t_{\max }(n)$ is the time at which the $n$th bubble reaches its maximal radius, $\gamma$ is the fluid surface tension, and $R_{b}(t)$ and $\dot{R}_{b}(t)$ represent the instantaneous radius and velocity of the $n$th bubble. Along with bubble growth, a fraction of the energy flowing to the fluid is converted to acoustic energy. The corresponding expression is

$$
E_{\mathrm{a}}(n)=\int_{\Delta t_{\text {wave }}(n)} \int_{S} e_{\mathrm{a}} c_{S} d S d t,
$$

where $\Delta t_{\text {wave }}(n)$ is the time during which the wave generated by nanobubble growth travels through a close surface $S$ at $r=$ $R_{\mathrm{np}}$, and $e_{\mathrm{a}}=\Delta P^{2} / 2 \rho c_{s}^{2}$ is the acoustic energy density, with $c_{s}$ the wave celerity and $\rho$ the fluid density.

\section{APPENDIX B}

Here we provide a justification for two expressions of the particle surface temperature used in the text. For notational convenience, let us introduce two dimensionless parameters, $\gamma$ and $\beta$, defined from

$$
\gamma^{2}=\frac{C_{\mathrm{np}} D}{3 G R_{\mathrm{np}}}, \quad \beta^{2}=\frac{C_{\mathrm{np}} G R_{\mathrm{np}}}{3 C k} .
$$

We also denote the Kapitza time as $\tau_{\mathrm{K}}=C R_{\mathrm{np}} / 3 G$. For a nanoparticle with radius $R_{\mathrm{np}}=50 \mathrm{~nm}$ and the parameters considered in the text, $\gamma$ is small and $\beta$ typically around unity (0.16 and 1.2 , respectively).

\section{Maximum particle surface temperature for low medium heating}

We consider heat diffusion in the medium surrounding a spherical nanoparticle, whose temperature is assumed to be uniform. Taking $\tau_{\mathrm{K}}$ as the unit time and $R_{\mathrm{np}}$ as the unit length, the governing equations in dimensionless form are

$$
\partial_{t} f_{\mathrm{np}}=-\left(f_{\mathrm{np}}-f_{\mathrm{s}}\right), \quad \partial_{t} f=\gamma^{2} \Delta_{r} f,
$$

together with the boundary and initial conditions

$$
\begin{aligned}
& -\gamma \partial_{r} f=\beta\left(f_{\mathrm{np}}-f_{\mathrm{s}}\right) \quad \text { for } \quad r=1 \text {, } \\
& f_{\mathrm{np}}=1, f=0 \text { for } t=0 .
\end{aligned}
$$

Here, $f_{\mathrm{np}}(t)$ is the particle temperature, $f(t, r)$ the medium temperature, and $f_{\mathrm{s}}(t)=f(t, 1)$ the medium temperature at the particle surface. $\Delta_{r}$ is the the radial part of the Laplacian. The initial temperature is unity in the particle and 0 elsewhere [77]. Note that because this condition is equivalent to a situation where the particle is impulsively heated at the initial time, the solution can be seen as a Green function for the problem.

We consider the limiting case $\gamma \rightarrow 0$ with the ratio $\beta / \gamma$ kept fixed. Physically, such a situation is reached for a highly conductive medium and implies a medium temperature remaining significantly below the particle temperature $\left(f, f_{\mathrm{s}} \ll\right.$ $\left.f_{\mathrm{np}}\right)$. We solve the equations perturbatively in $\gamma$, focusing on the lowest order only. Equation (B2) for the particle temperature immediately leads to $f_{\mathrm{np}}(t)=e^{-t}$. As regards the medium temperature, we use a Laplace transform with respect to time $(t \rightarrow s)$ to solve the coupled equations. The Laplace transform of the surface temperature is found to be $\tilde{f}_{\mathrm{s}}(s)=$ $\beta \tilde{f}_{\mathrm{np}}(s) / \sqrt{s}$, which in the time domain yields the relation

$$
f_{\mathrm{s}}(t)=\beta \int_{0}^{t} \frac{f_{\mathrm{np}}\left(t-t^{\prime}\right)}{\sqrt{\pi t^{\prime}}} d t^{\prime} .
$$

Using the solution for $f_{\mathrm{np}}(t)$ and performing the integration gives

$$
f_{\mathrm{s}}(t)=\frac{2 \beta}{\sqrt{\pi}} F(\sqrt{t}),
$$

with $F$ the Dawson function. Returning to the variables used in the text, an approximation for the maximal temperature 
$T_{\max }$ reached at the particle surface is then

$$
T_{\max }-T_{0}=\frac{3 F_{\max }^{D}}{2 \pi^{3 / 2}} \beta \frac{\zeta F}{C_{\mathrm{np}}},
$$

where $F_{\max }^{D} \approx 0.541$ is the maximum of the Dawson function. This expression is used to derive Eq. (16) in the text.

\section{Short-time behavior of the surface temperature}

Here we make no assumption about the value of the $\gamma$ and $\beta$ parameters. Our starting point is the explicit expression for the particle temperature given by Carslaw and Jaeger [78]. Using our notation it reads

$$
f_{\mathrm{np}}(t)=\frac{2 \beta \gamma}{\pi} \int_{0}^{\infty} \frac{u^{2} e^{-\gamma^{2} u^{2} t} d u}{\left[\gamma(\gamma+\beta) u^{2}-1\right]^{2}+\left[\gamma^{2} u^{3}-u\right]^{2}} .
$$

Now, using Eq. (B2) for $f_{\text {np }}$ and a change of variable $v=$ $\gamma^{2} u^{2}$, one can write for the time derivative of the surface temperature

$$
\begin{aligned}
& f_{\mathrm{s}}^{\prime}(t)=\frac{\beta}{\pi} \int_{0}^{\infty} e^{-t v} \mathcal{F}(v) d v, \\
& \mathcal{F}(v)=\frac{v(v-1)}{[\gamma(\gamma+\beta) v-1]^{2}+v(v-1)^{2}} .
\end{aligned}
$$

We considered $f_{\mathrm{s}}^{\prime}(t)$ so that we can directly apply a Tauberian for Laplace transforms. Indeed, the integral above is the Laplace transform of function $\mathcal{F}(v)$ taken at value $t$ and denoted $\tilde{\mathcal{F}}(t)$ [79]. Now, by Tauberian theorem [80], $\mathcal{F}(v) \simeq$ $v^{-1 / 2}$ for $v \rightarrow \infty$ implies $\tilde{\mathcal{F}}(t) \simeq \sqrt{\pi} t^{-1 / 2}$ for $t \rightarrow 0$. After time integration, we thus obtain for the short-time behavior of the particle surface temperature

$$
f_{\mathrm{s}}(t) \simeq \frac{2 \beta}{\sqrt{\pi}} t^{1 / 2}
$$

Note that because $F(u) \simeq u$ for a small argument, the particular case of Eq. (B5) is consistent with the general behavior given by Eq. (B9). For a heating pulse of unit magnitude starting at $t=0$ and remaining constant afterwards, it suffices to integrate the Green function over time, giving in this case

$$
f_{\mathrm{s}}(t) \simeq \frac{4 \beta}{3 \sqrt{\pi}} t^{3 / 2} .
$$

Returning to variables with dimension, the short-time behavior of the surface temperature $T(t)$ for a long pulse is at leading order

$$
T(t)-T_{0}=\frac{\beta}{\pi^{3 / 2}}\left(\frac{t}{\tau_{\mathrm{K}}}\right)^{3 / 2} \frac{\zeta F}{C_{\mathrm{np}}},
$$

which is used to derive Eq. (23).
[1] E. Boulais, R. Lachaine, A. Hatef, and M. Meunier, Plasmonics for pulsed-laser cell nanosurgery: Fundamentals and applications, J. Photochem. Photobiol. C 17, 26 (2013).

[2] G. Baffou, F. Cichos, and R. Quidant, Applications and challenges of thermoplasmonics, Nat. Mater. 19, 946 (2020)

[3] C. M. Pitsillides, E. K. Joe, X. Wei, R. R. Anderson, and C. P. Lin, Selective cell targeting with light-absorbing microparticles and nanoparticles, Biophys. J. 84, 4023 (2003).

[4] G. Baffou, Thermoplasmonics: Heating Metal Nanoparticles Using Light (Cambridge University Press, Cambridge, UK, 2018).

[5] G. Baffou and R. Quidant, Thermo-plasmonics: Using metallic nanostructures as nano-sources of heat, Laser Photon. Rev. 7, 171 (2013).

[6] J. Lombard, T. Biben, and S. Merabia, Kinetics of Nanobubble Generation Around Overheated Nanoparticles, Phys. Rev. Lett. 112, 105701 (2014).

[7] K. Metwally, S. Mensah, and G. Baffou, Fluence threshold for photothermal bubble generation using plasmonic nanoparticles, J. Phys. Chem. C 119, 28586 (2015).

[8] A. Vogel, J. Noack, G. Hüttman, and G. Paltauf, Mechanisms of femtosecond laser nanosurgery of cells and tissues, Appl. Phys. B 81, 1015 (2005).

[9] J. E. Shepherd and B. Sturtevant, Rapid evaporation at the superheat limit, J. Fluid Mech. 121, 379 (1982).

[10] S. Glod, D. Poulikakos, Z. Zhao, and G. Yadigaroglu, An investigation of microscale explosive vaporization of water on an ultrathin Pt wire, Int. J. Heat Mass Transfer 45, 367 (2002).

[11] Z. Zhao, S. Glod, and D. Poulikakos, Pressure and power generation during explosive vaporization on a thin-film microheater, Int. J. Heat Mass Transfer 43, 281 (2000).
[12] V. P. Carey, Liquid-Vapor Phase-Change Phenomena: An Introduction to the Thermophysics of Vaporization and Condensation Processes in Heat Transfer Equipment (Taylor and Francis, New York, 2008).

[13] H. Löwen and P. A. Madden, A microscopic mechanism for shock-wave generation in pulsed-laser-heated colloidal suspensions, J. Chem. Phys. 97, 8760 (1992).

[14] L. Hou, M. Yorulmaz, N. R. Verhart, and M. Orrit, Explosive formation and dynamics of vapor nanobubbles around a continuously heated gold nanosphere, New J. Phys. 17, 013050 (2015).

[15] Y. Wang, M. E. Zaytsev, G. Lajoinie, H. L. The, J. C. T. Eijkel, A. van den Berg, M. Versluis, B. M. Weckhuysen, X. Zhang, H. J. W. Zandvliet, and D. Lohse, Giant and explosive plasmonic bubbles by delayed nucleation, Proc. Natl. Acad. Sci. USA 115, 7676 (2018).

[16] Y. Wang, M. E. Zaytsev, H. L. The, J. C. T. Eijkel, H. J. W. Zandvliet, X. Zhang, and D. Lohse, Vapor and gas-bubble growth dynamics around laser-irradiated, water immersed plasmonic nanoparticles, ACS Nano 11, 2045 (2017).

[17] T. Jollans and M. Orrit, Explosive, oscillatory, and Leidenfrost boiling at the nanoscale, Phys. Rev. E 99, 063110 (2019).

[18] J. Lombard, T. Biben, and S. Merabia, Ballistic heat transport in laser generated nano-bubbles, Nanoscale 8, 14870 (2016).

[19] J. Lombard, T. Biben, and S. Merabia, Threshold for vapor nanobubble generation around plasmonic nanoparticles, J. Phys. Chem. C 121, 15402 (2017).

[20] D. Wang, P. Cheng, and X. Quan, Photothermal nanobubble nucleation on a plasmonic nanoparticle: A 3D lattice Boltzmann simulation, Int. J. Heat Mass Transf. 140, 786 (2019).

[21] A. M. Fales, W. C. Vogt, K. A. Wear, T. J. Pfefer, and I. K. Ilev, Experimental investigation of parameters influencing plasmonic 
nanoparticle-mediated bubble generation with nanosecond laser pulses, J. Biomed. Opt. 24, 065003 (2019).

[22] M. O. Ogunyankin, J. E. Shin, D. O. Lapotko, V. E. Ferry, and J. A. Zasadzinski, Optimizing the NIR fluence threshold for nanobubble generation by controlled synthesis of 10-40 nm hollow gold nanoshells, Adv. Funct. Mater 28, 1705272 (2018).

[23] S. Wang, L. Fu, Y. Zhang, J. Wang, and Z. Zhang, Quantitative evaluation and optimization of photothermal bubble generation around overheated nanoparticles excited by pulsed lasers, J. Phys. Chem. C 122, 24421 (2018).

[24] C. P. Lin and M. W. Kelly, Cavitation and acoustic emission around laser-heated microparticles, Appl. Phys. Lett. 72, 2800 (1998).

[25] N. Kavokine, S. Zou, R. Liu, A. Niguès, B. Zou, and L. Bocquet, Ultrafast photomechanical transduction through thermophoretic implosion, Nat. Commun. 11, 50 (2020).

[26] A. N. Volkov, C. Sevilla, and L. V. Zhigilei, Numerical modeling of short pulse laser interaction with $\mathrm{Au}$ nanoparticle surrounded by water, Appl. Surf. Sci. 253, 6394 (2007).

[27] E.-A. Brujan, Stress wave emission from plasmonic nanobubbles, J. Phys. D: Appl. Phys. 50, 015304 (2016).

[28] V. K. Pustovalov, A. S. Smetannikov, and V. P. Zharov, Photothermal and accompanied phenomena of selective nanophotothermolysis with gold nanoparticles and laser pulses, Laser Phys. Lett. 5, 775 (2008).

[29] V. P. Zharov, R. R. Letfullin, and E. N. Galitovskaya, Microbubbles-overlapping mode for laser killing of cancer cells with absorbing nanoparticle clusters, J. Phys. D: Appl. Phys. 38, 2571 (2005).

[30] V. P. Zharov, K. E. Mercer, E. N. Galitovskaya, and M. S. Smeltzer, Photothermal nanotherapeutics and nanodiagnostics for selective killing of bacteria targeted with gold nanoparticles, Biophys. J. 90, 619 (2006).

[31] D. O. Lapotko, E. Lukianova, and A. A. Oraevsky, Selective laser nano-thermolysis of human leukemia cells with microbubbles generated around clusters of gold nanoparticles, Lasers Surg. Med. 38, 631 (2006).

[32] D. Wen, Intracellular hyperthermia: Nanobubbles and their biomedical applications, Int. J. Hyperthermia 25, 533 (2009).

[33] K. R. Rau, P. A. Quinto-Su, A. N. Hellman, and V. Venugopalan, Pulsed laser microbeam-induced cell lysis: Time-resolved imaging and analysis of hydrodynamic effects, Biophys. J. 91, 317 (2006).

[34] C. Yao, F. Rudnitziki, Y. He, Z. Zhang, G. Huttmann, and R. Rahmanzadeh, Cancer cell-specific protein delivery by optoporation with laser-irradiated gold nanorods, J. Biophoton. 13, e20200017 (2020).

[35] H. Ju, R. A. Roy, and T. W. Murray, Gold nanoparticle targeted photoacoustic cavitation for potential deep tissue imaging and therapy, Biomed. Opt. Express 4, 66 (2013).

[36] M. Sarimollaoglu, D. A. Nedosekin, Y. A. Menyaev, M. A. Juratli, and V. P. Zharov, Nonlinear photoacoustic signal amplification from single targets in absorption background, Photoacoustics 2, 1 (2014).

[37] S. Wang, L. Fu, J. Xin, S. Wang, C. Yao, Z. Zhang, and J. Wang, Photoacoustic response induced by nanoparticle-mediated photothermal bubbles beyond the thermal expansion for potential theranostics, J. Biomed. Opt. 23, 125002 (2018).
[38] I. G. Calasso, W. Craig, and G. J. Diebold, Photoacoustic Point Source, Phys. Rev. Lett. 86, 3550 (2001).

[39] A. Prost, F. Poisson, and E. Bossy, Photoacoustic generation by a gold nanosphere: From linear to nonlinear thermoelastics in the long-pulse illumination regime, Phys. Rev. B 92, 115450 (2015).

[40] T. Grosges and D. Barchiesi, Numerical study of photoacoustic pressure for cancer therapy, Appl. Sci. 6, 357 (2016).

[41] L. Cavigli, A. Milanesi, B. N. Khlebtsov, S. Centi, F. Ratto, N. G. Khlebtsov, and R. Pini, Impact of Kapitza resistance on the stability and efficiency of photoacoustic conversion from gold nanorods, J. Colloid Interface Sci. 578, 358 (2020).

[42] J. M. Sun, B. S. Gerstman, and B. Li, Bubble dynamics and shock waves generated by laser absorption of a photoacoustic sphere, J. Appl. Phys. 88, 2352 (2000).

[43] M. G. González, X. Liu, R. Niessner, and C. Haisch, Strong size-dependent photoacoustic effect on gold nanoparticles by laser-induced nanobubbles, Appl. Phys. Lett. 96, 174104 (2010).

[44] S.-B. Wen, K. J. Ly, A. Bhaskar, M. S. Schmidt, and R. J. Thomas, Direct numerical simulation of the initial stage of a thermally induced microcavitation in a water-rich biotissue triggered by a nanosecond pulsed laser, J. Biomed. Opt. 22, 056002 (2017).

[45] Y. Shi, S. Yang, and D. Xing, New insight into photoacoustic conversion efficiency by plasmon-mediated nanocavitation: Implications for precision theranostics, Nano Res. 10, 2800 (2017).

[46] R. Gao, R. Fu, W. Jiao, G. Fan, C. Liang, J. Chen, H. Ren, Y. Wang, W. Liu, S. Ren, X. Ren, Q. Wei, and M. Sun, Optoacoustic effect of Au nanoparticles in water under irradiation of pulse laser, Optik 202, 163512 (2020).

[47] E. Faraggi, B. S. Gerstman, and J. Sun, Biophysical effects of pulsed lasers in the retina and other tissues containing strongly absorbing particles: Shockwave and explosive bubble generation, J. Biomed. Opt. 10, 064029 (2005).

[48] J. Lombard, T. Biben, and S. Merabia, Nanobubbles around plasmonic nanoparticles: Thermodynamic analysis, Phys. Rev. E 91, 043007 (2015).

[49] A. Onuki, Dynamic Van Der Waals Theory of Two-Phase Fluids in Heat Flow, Phys. Rev. Lett. 94, 054501 (2005).

[50] H. Movahedinejad and H. Nadjari, Temperature variations of gold nanoparticle and dynamics of plasmonic bubble in water under nanosecond pulsed laser, Plasmonics 15, 631 (2019).

[51] F. Magaletti, L. Marino, and C. Casciola, Shock Wave Formation in the Collapse of a Vapor Nanobubble, Phys. Rev. Lett. 114, 064501 (2015).

[52] F. Magaletti, M. Gallo, L. Marino, and C. M. Casciola, Shockinduced collapse of a vapor nanobubble near solid boundaries, Int. J. Multiphase Flow 84, 34 (2016).

[53] K. Sasikumar and P. Keblinski, Molecular dynamics investigation of nanoscale cavitation dynamics, J. Chem. Phys. 141, 234508 (2014)

[54] J. H. Pu, W. Wang, and W. S. Wang, Generation and evolution of nanobubble on heated nanoparticles: A molecular dynamics study, Langmuir 36, 2375 (2020).

[55] V. M. Alipchenkov, L. A. Dombrovski, and L. I. Zaichik, The growth and stability of vapor film on the surface of a hot spherical particle, High Temperature 40, 100 (2002). 
[56] C. F. Bohren and D. R. Huffman, Absorption and Scattering of Light by Small Particles (Wiley-VCH Verlag GmbH, Weinheim, 1998).

[57] J.-L. Barrat and F. Chiaruttini, Kapitza resistance at the liquidsolid interface, Mol. Phys. 101, 1605 (2003).

[58] S. Merabia, S. Shenogin, L. Joly, P. Keblinski, and J.-L. Barrat, Heat transfer from nanoparticles: A corresponding state analysis, Proc. Natl. Acad. Sci. USA 106, 15113 (2009).

[59] S. Merabia, P. Keblinski, L. Joly, L. J. Lewis, and J.-L. Barrat, Critical heat flux around strongly heated nanoparticles, Phys. Rev. E 79, 021404 (2009).

[60] D. M. Anderson, G. B. McFadden, and A. A. Wheeler, Diffuseinterface methods in fluid mechanics, Annu. Rev. Fluid Mech. 30, 139 (1998).

[61] A. Onuki, Dynamic van der Waals theory, Phys. Rev. E 75, 036304 (2007).

[62] J. S. Rowlinson and B. Widom, Molecular Theory of Capillarity (Courier Corporation, North Chelmsford, MA, 2002).

[63] K. S. Schweizer and E. J. Saltzman, Activated hopping, barrier fluctuations, and heterogeneity in glassy suspensions and liquids, J. Phys. Chem. B 108, 19729 (2004).

[64] A. J. Barlow and E. Yazgan, Pressure dependence of the velocity of sound in water as a function of temperature, Br. J. Appl. Phys. B 18, 645 (1967).

[65] A. G. Doukas and T. J. Flotte, Physical characteristics and biological effects of laser-induced stress waves, Ultrasound Med. Biol. 22, 151 (1996).

[66] A. Vogel, S. Busch, and M. Asiyo-Vogel, Time-resolved measurements of shock-wave emission and cavitation-bubble generation in intraocular laser surgery with ps- and ns-pulses and related tissue effects, in Ophthalmic Technologies III, Vol. 1877, edited by J.-M. A. Parel and Q. Ren (SPIE, Bellingham, WA, 1993), pp. 312-322.

[67] C. Yao, R. Rahmanzadeh, E. Endl, Z. Zhang, J. Gerdes, and G. Hüttmann, Elevation of plasma membrane permeability by laser irradiation of selectively bound nanoparticles, J. Biomed. Opt. 10, 064012 (2005).

[68] M. Kitz, S. Preisser, A. Wetterwald, M. Jaeger, G. N. Thalmann, and M. Frenz, Vapor bubble generation around gold nanoparticles and its application to damaging of cells, Biomed. Opt. Express 2, 291 (2011).

[69] V. P. Zharov, V. Galitovsky, and M. Viegas, Photothermal detection of local thermal effects during selective nanophotothermolysis, Appl. Phys. Lett. 83, 4897 (2003).

[70] The difference in nanoparticle radius, i.e., $R_{\mathrm{np}}=50 \mathrm{~nm}$ vs $R_{\text {np }}=40 \mathrm{~nm}$, cannot explain the gap between the simulation results and the Rayleigh-Plesset predictions, as for short pulses the pressure amplitude is found to scale as $R_{\mathrm{np}}^{1.5}$ as shown in Fig. 6.

[71] Recall that, as shown in Fig. 5, there are many echoes associated with all the nanobubble rebounds, which have comparable amplitudes.

[72] Denoting as $k$ the thermal conductivity of the fluid, the Kapitza length is $l_{K}=k / 3 G$.

[73] D. Boal, Mechanics of the Cell (Cambridge University Press, Cambridge, UK, 2012).

[74] A. G. Doukas, D. J. McAuliffe, S. Lee, V. Venugopalan, and T. J. Flotte, Physical factors involved in stress-wave-induced cell injury: The effect of stress gradient, Ultrasound Med. Biol. 21, 961 (1995).

[75] S. Lee, T. Anderson, H. Zhang, T. J. Flotte, and A. G. Doukas, Alteration of cell membrane by stress waves in vitro, Ultrasound Med. Biol. 22, 1285 (1996).

[76] Alternatively, one could consider the pressure spatial gradient.

[77] In all cases, only the difference with respect to the initial temperature is considered.

[78] H. Carslaw and J. Jaeger, Conduction of Heat in Solids (Oxford University Press, New York, 1959).

[79] Note that here the time $t$ is the Laplace variable and $v$ is the original variable.

[80] W. Feller, An Introduction to Probability Theory and Its Applications (John Wiley \& Sons, New York, 1968). 